\title{
Sex-specific glycosylation of secreted immunomodulatory proteins in the filarial nematode Brugia malayi
}

Joseph Koussa ${ }^{1,2,7}$, Burcu Vitrinel ${ }^{1}$, Peter Whitney ${ }^{1}$, Brian Kasper ${ }^{3}$, Lara K. Mahal ${ }^{3,4}$, Christine Vogel $^{1}$, Sara lustigman ${ }^{5}$, Kourosh Salehi-Ashtiani $^{2}$, Elodie Ghedin ${ }^{1,6,7 *}$

${ }^{1}$ Department of Biology, New York University and Center for Genomics and Systems Biology (CGSB), New York, NY, USA

${ }^{2}$ Division of Science and Math, New York University Abu Dhabi, Abu Dhabi, UAE

${ }^{3}$ Department of Chemistry, New York University, New York, NY, USA

${ }^{4}$ Department of Chemistry, University of Alberta, Edmonton, AB, CANADA

${ }^{5}$ Laboratory of Molecular Parasitology, Lindsley F. Kimball Research Institute, The New York Blood Center, New York, NY, USA

${ }^{6}$ Department of Epidemiology, School of Global Public Health, New York University, New York, NY, USA

${ }^{7}$ Systems Genomics Section, Laboratory of Parasitic Diseases, National Institute of Allergy and Infectious Diseases, National Institutes of Health, Bethesda, MD, USA

*Corresponding author: elodie.ghedin@nih.gov 


\section{Abstract}

2

3 The extended persistence of filarial nematodes within a host suggests immunomodulatory mechanisms that allow the parasites to resist or evade the host immune response. There is increasing evidence for immunomodulatory glycans expressed by a diversity of parasitic worms. In this study, we integrate multiple layers of the host-parasite interface to investigate the glycome of a model filarial parasite, Brugia malayi. We report a significant overrepresentation of terminal GalNAc moieties in adult female worms coupled with an overall upregulation in O-glycosylation, T-antigen expression, and a bias for galactose containing glycans. Adult males preferentially displayed a bias for terminal GlcNAc containing glycans, and fucosylated epitopes. Subsequent proteomic analysis confirmed sex-biases in protein glycosylation and highlighted the sex-specific glycosylation of well characterized immunomodulators expressed and secreted by B. malayi. We identify sex-specific effectors at that interface and suggest approaches to selectively interfere with the parasitic life cycle and potentially control transmission. 


\section{Introduction}

Brugia malayi is a parasitic nematode and, along with Wuchereria bancrofti, a causative agent of lymphatic filariasis in humans. The life cycle of $B$. malayi involves both a mosquito vector and a human host in which the worms can persist for up to eight years as adults colonizing the lymphatic system (Nutman). In contrast to the majority of nematodes, including the freeliving model nematode $C$. elegans, parasitic worms like $B$. malayi are sexually dimorphic, with male and female worms coexisting within the same host. Once sexual maturity is reached (>120 days post infection), fertilized adult females release microfilariae (mf)—the youngest life stage of the worm-into blood circulation. These are then picked up by the mosquito during a blood meal. In the mosquito, the mf develops into infective stage 3 larvae (L3), which can be transmitted to a new human host during a subsequent blood meal.

Sexual dimorphism in parasitic nematodes has been suggested to be a response to host immune pressure (Gemmill et al.). Sexual dimorphism has been well characterized in $B$. malayi (Michalski and Weil; Jiang, Li, et al.; Kashyap et al.). At both the transcriptomic and proteomic levels, adult male and female B. malayi display sex biases in gene expression with adult males characterized by enrichment for genes involved in energy production, metabolic processes and cytoskeletal proteins, while adult females have gene expression profiles enriched in signatures for RNA modification and transcription (Jiang, Malone, et al.). Proteomic analysis of sex-specific secretomes from B. malayi identified significant differences between adult male and female worms; $70 \%$ of proteins secreted by adult males and $65 \%$ secreted by adult females were unique to each sex (Bennuru et al.).

More recently, proteomic analysis of exosome-like vesicles secreted from adult B. malayi revealed sex-dependence in protein cargo. Adult female-secreted vesicles had a high content in Bma-Galectin-2, Triose Phosphate isomerase (TPI), Macrophage migration inhibitory factor (MIF1) and Thioredoxin peroxidase 2 among others, while the male-secreted vesicles were enriched for small GTPases, structural actin and tubulin, and a subset of heat shock proteins (Harischandra et al.). While sex differences in $B$. malayi have been investigated at both transcriptomic and proteomic levels, they remain understudied at the glycomic level. 
Well conserved across evolution, glycosylation is a dynamic, non-templated process, dependent on enzymatic and substrate availability and resulting in a diversity of carbohydrate structures. Glycosylation plays key roles in regulating diverse biological processes, ranging from signaling and immune activation to development and reproduction in eukaryotic organisms (Varki, Ajit; Cummings, R. D.; Esko, J. D.; Freeze, H. H.; Stanley, P.; Bertozzi, C. R.; Hart, G. W.; Etzler and E.). Glycosylation is also known to act as a checkpoint in the proper folding of proteins within secretory pathways, as a large portion of secreted proteins from eukaryotes are glycosylated. In helminthic infections, glycoconjugates are involved in the underlying immunomodulation of infected hosts (Prasanphanich et al.; Harn et al.; Van Vliet et al.; Khoo and Dell). Several such glycoconjugates are at the host-parasite interface. For example, parasite excreted/secreted (ES) glycoproteins were shown to actively modulate the host immune system by physically interacting with cell surface receptors on dendritic cells (DC) and macrophages (van den Berg et al.; Rodríguez et al.). Studies have shown that in $B$. malayi, intact glycan structures on secreted proteins are necessary for the induction of the characteristic Th2 immune responses (Tawill et al.). In the blood fluke S. mansoni, studies revealed significant sex differences in glycan structures where females mainly carried Gal $\beta 1$ 4GIcNAc (Type II LacNAc) and Galß1-4(Fuc 1 1-3)GIcNAc (LewisX) antennae structures, whereas in males GalNAc $\beta 1-4 G l c N A c$ (Lacdi-NAc; LDN) and GalNAc $\beta 1-4(F u c \alpha 1-3)$ GlcNAc (LDN-F) were prevalent in N-glycans, suggesting differential effects on host responses (Wuhrer et al.). Studies of the parasitic worms Fasciola hepatica, Oesophagostomum dentatum, and S. mansoni report sex and life stage biases in $\mathrm{N}$-glycosylation profiles (Rodríguez et al.; Jiménez-Castells et al.). However, little information exists with respect to Oglycosylation trends and an in-depth characterization of glycosylation in filarial nematodes is lacking. Unraveling the sex-specific glycocode in B. malayi not only furthers our understanding of the biology of sexes and parasitism in nematodes, but also uncovers a set of new targets 
101 for anti-helminthic therapies or vaccine development, with the unique feature of potentially

102 targeting male or female worms independently.

104 Herein we profile protein glycosylation in B. malayi using lectin microarray technology

105 (Pilobello, Slawek, et al.; Agrawal et al.; Heindel et al.) and combine that with an expanded 106 analysis of the sex-specific secretomes. We identified sex-dependent glycan and glycoprotein

107 expression. We focused on the identification of sex-specific differentially glycosylated proteins

108 at the host-parasite interface and evaluated their potential role in the immunomodulatory

109 arsenal of B. malayi. We report the differential glycosylation in male and female B. malayi of

110 three previously characterized immunomodulatory proteins, Bma-MIF-1 (Prieto-Lafuente et

111 al.), Bma-FAR-1(Zhan et al.) and Bma-IPGM-1 (Singh et al.). We also report the sex-

112 dependent glycosylation of two recently suggested drug targets, a phosphoglycerate kinase

113 (Bm13839) (Kumar et al.) and a Calumenin (Bm5089) (Choi et al.), along with a subset of

114 immune-relevant proteins, further suggesting sex-specific host-parasite interactions, that may

115 be moderated by glycans, in $B$. malayi infections. 


\section{Results}

\section{B. malayi adult females secrete a larger diversity of proteins than adult males}

123 Although sex-dependent gene expression in B. malayi worms has been well characterized

124 (Bennuru et al.; Hewitson et al.; Grote et al.; Moreno and Geary), the stage- and sex-specific secretomes remain only partially defined. To gain a more complete understanding of the $B$. malayi secretome, in light of updated genome annotations and updated chromosome assemblies (Fauver et al, Tracey et al, Ghedin et al), we collected ES proteins from worms

128 cultured in vitro at different life stages — mf, L3, L4, adult males, and adult females (Fig. 1A).

129 Label-free mass spectrometric analysis of these samples identified a total of 1,114 unique

130 proteins across life stages and sexes in the B. malayi secretome (Supp. Table S1a). These

131 included previously identified proteins as well as an additional 444 proteins. It is worth noting that $47 \%$ of all identified proteins have no associated known functions. A summary table detailing overlaps with all preceding B. malayi proteomic studies can be found in Supp. Table

134 S1b. We recovered $60 \%$ of proteins identified in (Mersha et al.) as GPI-anchored and $80 \%$ of 135 proteins from exosome-like vesicles (EV) secreted by both adult sexes (Harischandra et al.).

136 We also recovered $72 \%$ and $78 \%$ of proteins identified by Moreno et al and Hewiston et al, respectively, as part of B. malayi's secretome (Moreno and Geary; Hewitson et al.). However, we only overlapped with $18 \%$ of the proteins identified by Bennuru et al (Bennuru et al.) as part of the secretome. Our data indicate that $70 \%$ of detected proteins were shared by at least

140 two of the stages and that adult females secreted the largest diversity of proteins with 238

141 unique to this stage compared to 6 unique to adult males (Fig. 1B). 
A

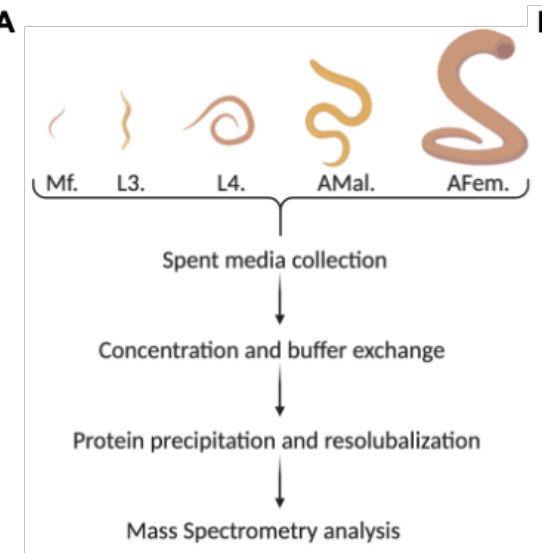

Mass Spectrometry analysis
B

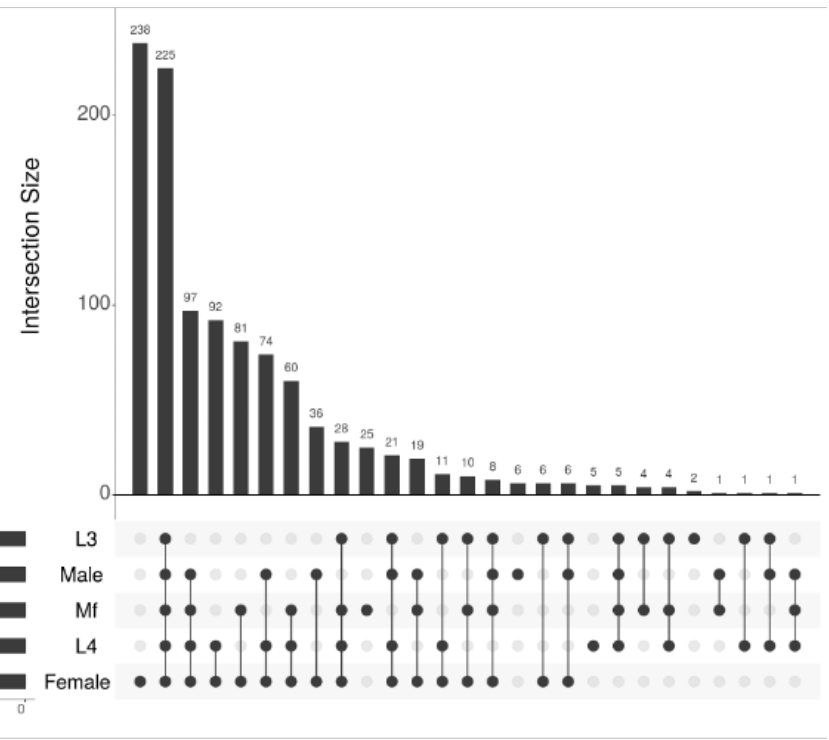

C

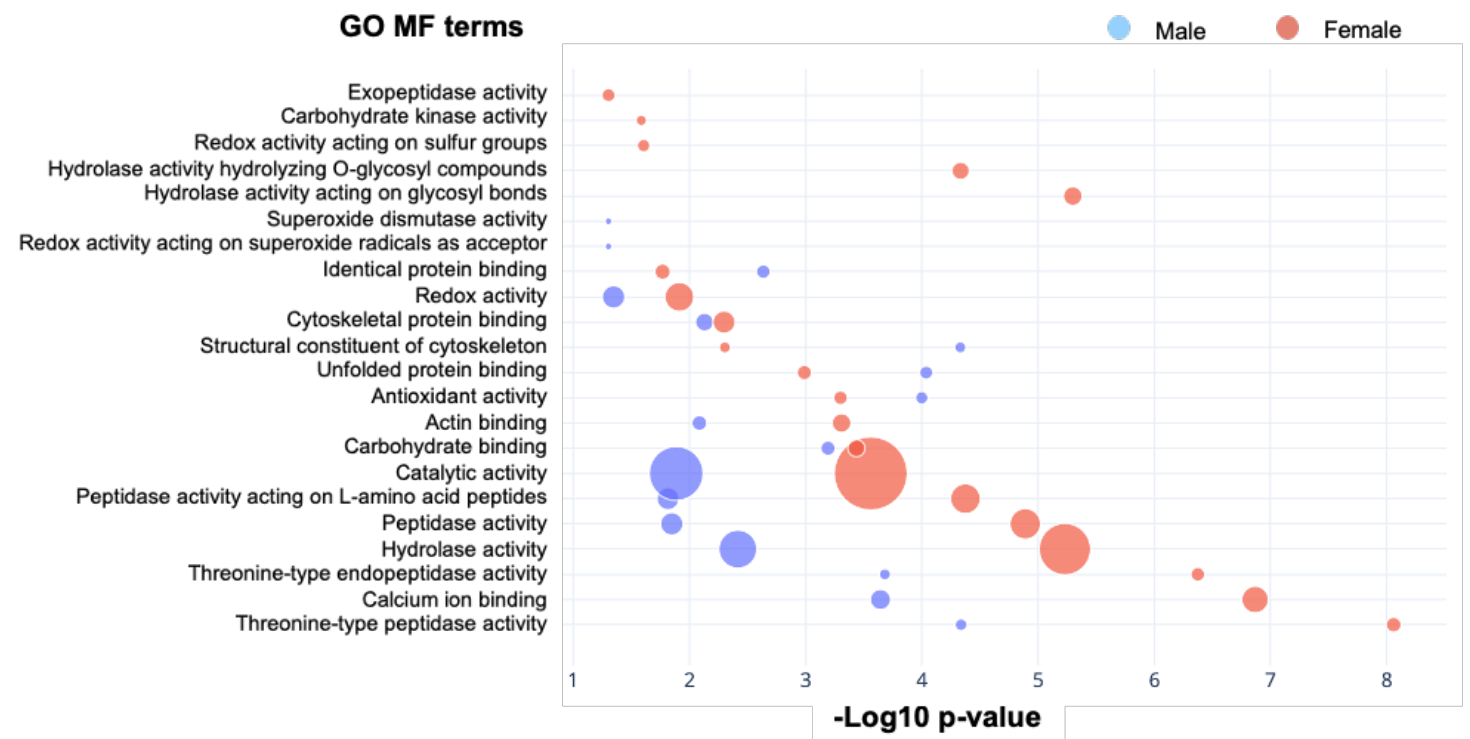

Figure 1: Proteomic characterization of stage- and sex-specific secretomes in B. malayi. A. Schematic representation of how B. malayi secretomes were collected for MS-MS analysis. B. UpsetR plot representing the intersections in stage- and sex-specific secretomes. The total number of proteins identified from each stage or sex are shown as set sizes. C. GO Molecular Function (MF) enrichment analysis of secreted proteins from adult males and females. Values shown represent -Log10 p-value and dot sizes are proportional to the number of proteins associated with each term.

144 To determine the functional implications of differentially secreted proteins, we performed

145 functional enrichment on the sex-dependent protein sets (Fig. 1C). In adult female worms, we

146 observed specific enrichment of hydrolases acting on glycosyl residues, carbohydrate kinases

147 and proteins involved in sulfur redox reactions ( $p$-values $<0.05)$, while in adult males we see

148 a significant enrichment of proteins involved in superoxide redox reactions and superoxide 
dismutase activities ( $p$-values < 0.05). Male and female adult $B$. malayi secrete a large number of proteins with catalytic activity (e.g. threonine-type endopeptidases) and carbohydrate binding proteins, both of which are associated with helminth immunomodulatory properties

152 (McSorley et al.).

\section{B. malayi has a glycogenome conserved across the filariae.}

As observed for most eukaryotic organisms, helminths glycosylate secreted proteins. These glycoproteins play key roles in immune function, and glycosylation is crucial to their activity

159 (Cvetkovic et al.; Ahmed et al.). It is therefore important to define glycan structures decorating 160 secreted proteins at the host-parasite interface. Determining the diversity of glycan structures expressed by an organism is largely dictated by the nature and number of glycosylationrelated enzymes and available substrates. To construct a comprehensive list of glycosylation enzymes, we interrogated the $B$. malayi genome using a series of functional annotations.

164 Gene Ontology (GO) and KEGG annotations for genes and pathways were used to assign 165 glycosylation-related proteins. We identified 116 genes with an associated GO term relevant 166 to protein glycosylation and 143 genes assigned to KEGG glycosylation pathways. A full

167 BLASTp search of the B. malayi proteome against a "human glycosylation proteome" 168 uncovered 136 genes with significant similarities. A full genome Hidden Markov Model scan 169 for protein families (Pfam) was done to complement the analysis, uncovering 112 genes with 170 Pfam annotations relevant to protein glycosylation. Overall, 285 genes were identified and were assigned to the B. malayi glycogenome (Fig. 2A; Supp. Table S2a). 
172 We evaluated the conservation of glycosylation across different clades of worms by

173 comparative analysis of glycosylation-related orthologs in other helminth genomes. The

174 analysis reveals a high degree of conservation within filarial nematodes (Fig. 2B, VII-XII) with

175 around $56 \%$ of the genes conserved at $>50 \%$ similarity. The data also indicated clear

176 divergence from blood and liver flukes (Fig. 2B, V-VI) with $33 \%$ of the genes having no

177 orthologs, and an intermediate profile of conservation when compared to hookworms,

178 whipworms or the free-living nematode C. elegans with only $14 \%$ of the genes having no

179 orthologs (Fig. 2B, I, IV, and II, respectively). Overall, the data show a higher conservation

180 and similarity in the glycogenomes of filarial parasites and class III nematodes, and a clear

181 divergence from trematodes such as F. hepatica and S. mansoni.

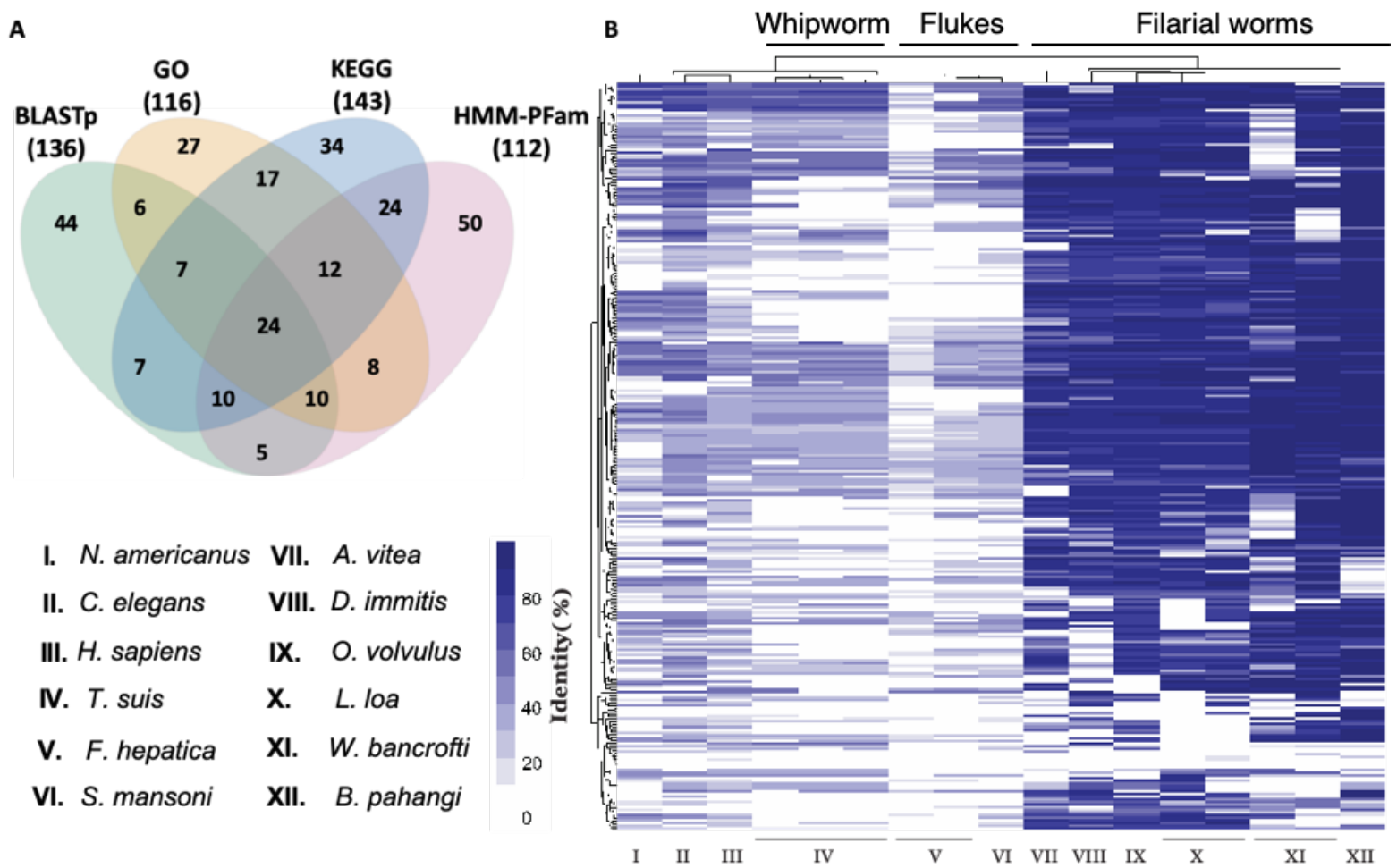

Figure 2: B. malayi's glycogenome and ortholog analysis of glycosylation related genes across other worm genomes, and the human genome. A. Venn diagram representing the overlap analysis of the different functional annotation approaches used to identify glycosylation related genes in $B$. malayi. B. Ortholog analysis of all B. malayi glycosylation genes in parasitic and non-parasitic worms. Data shown represent the percentage in genetic identity between $B$. malayi glycosylation related genes and their corresponding orthologues in other organisms. 

female adult worms

To investigate whether expression of the glycogenome is sex-dependent, we analyzed the transcriptome in adult male and female B. malayi. The overall expression profiles show high similarities between the sexes, as previously observed (Grote et al.). However, approximately $43 \%$ of the 285 glycosylation-related genes were significantly differentially expressed ( $p$-value

$191<0.05)$. The majority of these genes belong to $O$-and $N$-glycan-related biosynthetic pathways.

192 Female B. malayi significantly upregulate 50 glycosylation-related genes while males upregulate 72 (Supp. Table S2b). Mapping these results onto the biosynthetic pathways suggests higher expression of core 2/6 O-glycans in male worms (Fig. 3A). The results also indicate sex-dependent expression of specific fucose type O-glycans, with males having higher levels of GIcNAc-Fuc-(ser/thr), while females modify this epitope further to form predominantly Gal-GlcNAc-Fuc-(ser/thr) (Fig. 3B).

199 Females also show a higher expression of a protein-O-acetylglucosamine transferase (Bm4815), leading to the uncommon GlcNAc O-linked glycan epitope (Fig. 3C). Similarly, the mannose type O-glycan biosynthetic pathway shows male-biased expression of core M1 and core M2 glycans (Fig. 3D). Sex-dependent expression profiles are also apparent for $\mathrm{N}$-type glycan biosynthetic pathways where males display a higher expression of 17 genes within the $\mathrm{N}$-type glycosylation pathway as compared to 7 genes with higher expression in females; most are $\mathrm{N}$-glycan precursor and trimming enzymes. Both sexes also express genes related to glycan degradation, such as mannosidases and glycosidases, primarily involved in oligomannose and paucimannose N-glycan biosynthetic pathways (Supp. Table S2c). 
A

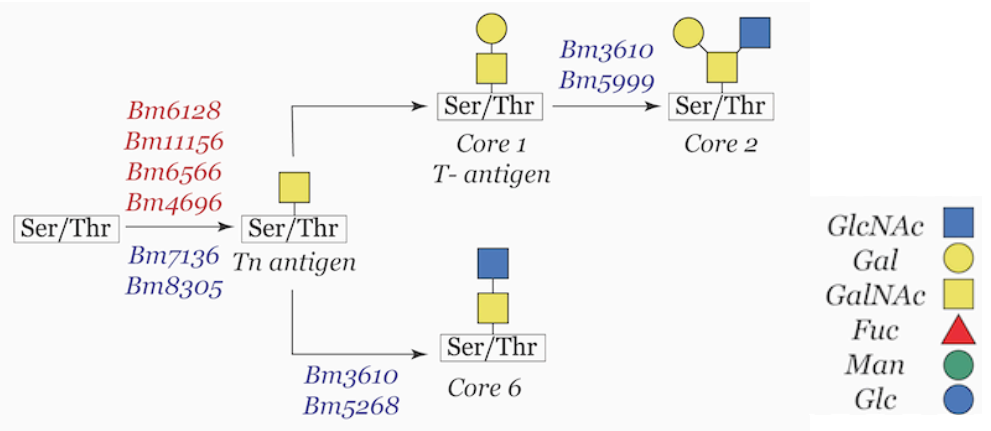

B

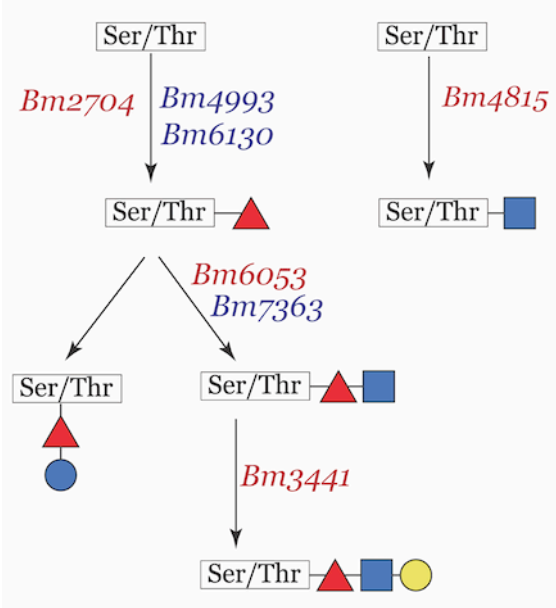

D

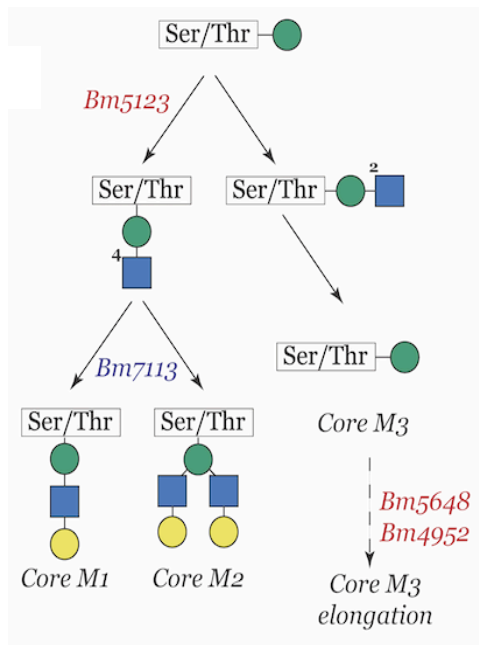

Figure 3: Differential expression of O-glycosylation related genes in adult male and female B. malayi. A. Partial representation of the mucin-type O-glycans biosynthetic pathway. B. malayi genes coding for enzymes catalyzing reactions are shown and color-coded. B. Fucose type $O$ glycans biosynthetic pathway and corresponding $B$. malayi genes color-coded by upregulation status. C. N-acetylglucosamine type O-glycans biosynthetic pathway and corresponding B. malayi genes color-coded by upregulation status. $\mathbf{D}$. Partial representation of the mannose type O-glycans biosynthetic pathway and corresponding $B$. malayi genes color-coded by upregulation status. Upregulated genes in females are highlighted in red and those upregulated in males are highlighted in blue.

211 Glycan epitope expression is sex-biased in B. malayi

213 The study of glycosylation in B. malayi has primarily focused on GPI-anchored glycoproteins

214 and staining of worms with specific lectins (Mersha et al.; Schraermeyer et al.; Kaushal et al.).

215 A more general glycomic analysis of B. malayi has not been performed. To confirm whether

216 the observed sex-biased differential expression of the $B$. malayi glycogenome translates into

217 sex-biased display of glycan epitopes, we analyzed whole worm lysates on lectin microarrays 
218 (Pilobello, Krishnamoorthy, et al.; Propheter et al.). This technology uses the known glycan

219 binding specificities of lectins to provide an epitope-specific readout of glycosylation. We

220 observed significant differences in glycosylation profiles between male and female worms

221 (Fig. 4). Female worms showed higher levels of terminal Gal/GalNAc epitopes, including core

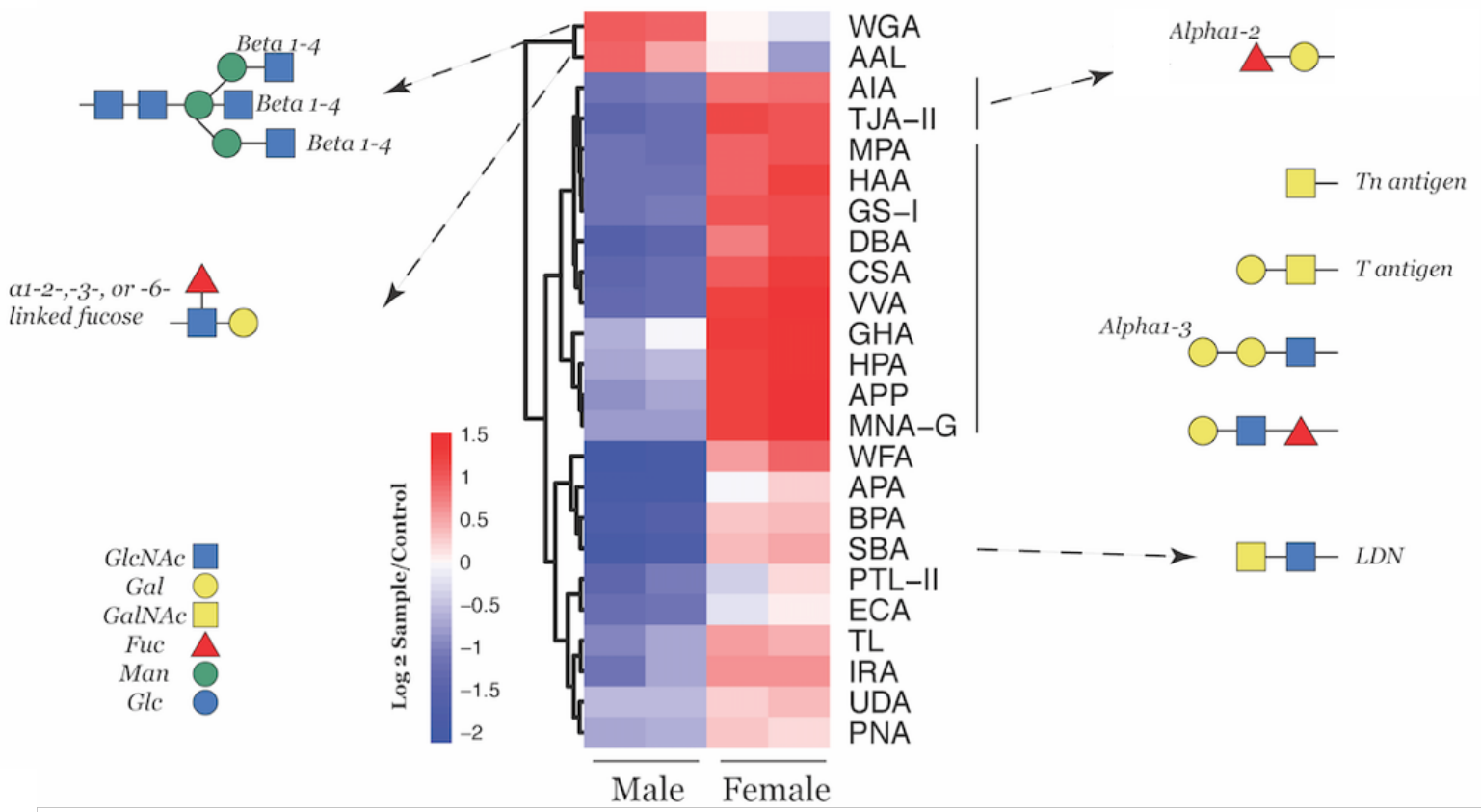

Figure 4: Glycomic profiling of $B$. malayi as determined by glycan binding to lectin arrays. Heatmap summarizing lectin array data comparing male and female glycans and representing significantly differentially displayed glycan epitopes between sexes $(p<0.05)$. Data shown represent Log2 of the fluorescence ratios between each sample and the control. Lectins are grouped by their corresponding recognition epitopes and representative glycan structure displaying respective epitopes are shown.

We suspected that biases in glycan epitopes would be associated with differential distribution within tissues in male and female worms, especially in the organs involved in secretion and excretion. To test this, we determined the spatial localization of fucosylated and/or 
231 worms with both fluorescently tagged AAL lectin, which binds fucosylated LacNAc (enriched

232 in males) and GS-I lectin, which binds to $\alpha$-galactose residues (enriched in females). We

233 observed a clear localization of fucosylated and galactosylated epitopes within the mouth and

234 cephalic alae of both males and females consistent with the known distribution of sensory

235 organs (Fig. 5A). Females showed a diffuse body staining with galactose-specific lectin GS-I,

236 with higher levels at the cuticular areas and within the ovaries, compared to a much lower

237 intensity of galactose staining across the body of male worms. In contrast, males displayed a

238 very specific and high intensity fluorescence of fucosylated residues in reproductive organs

239 and tissues (Fig. 5B) coupled to higher levels of fucosylated residues at the mouth and in the

240 cephalic regions. 
A

Male

head
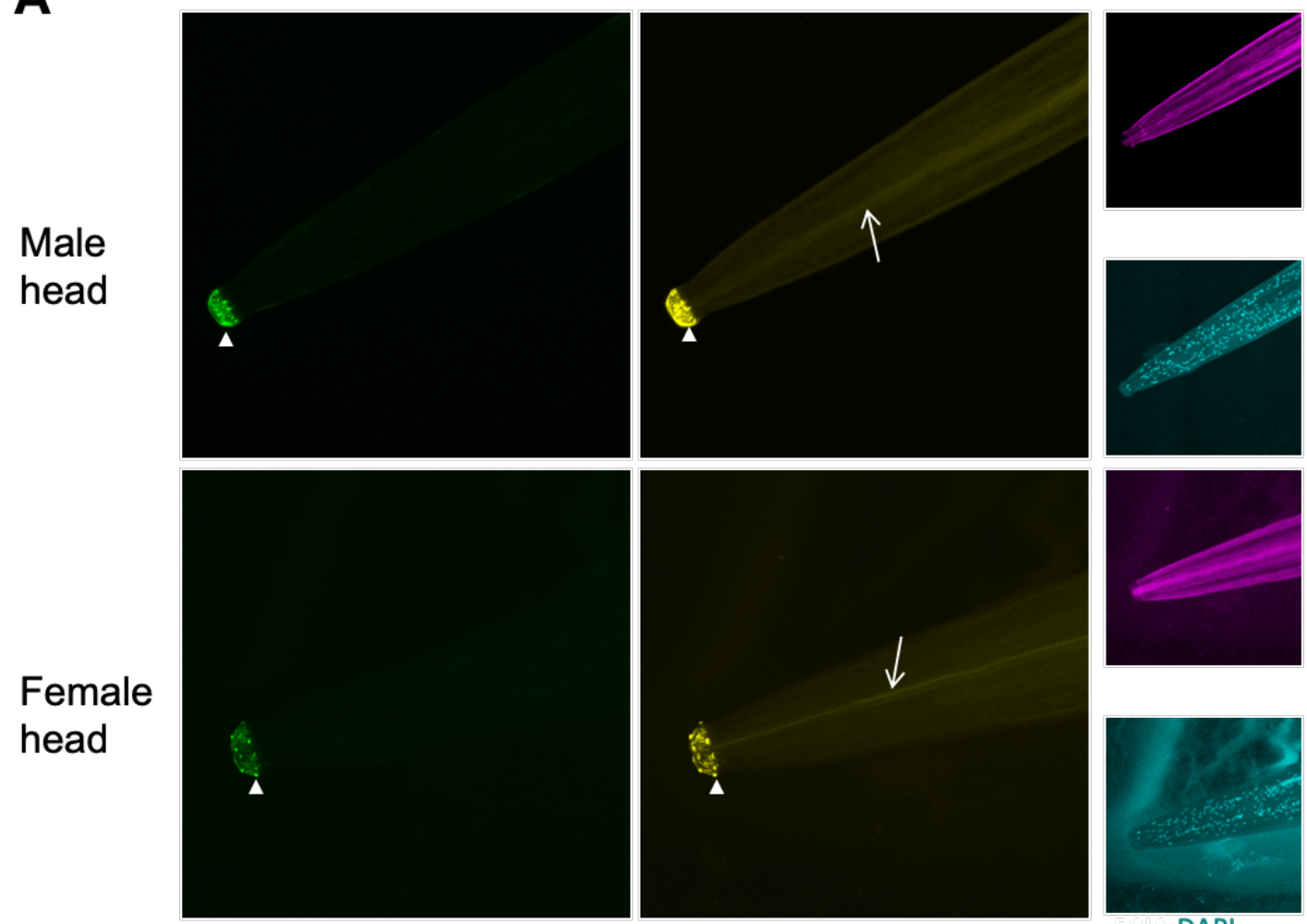

B
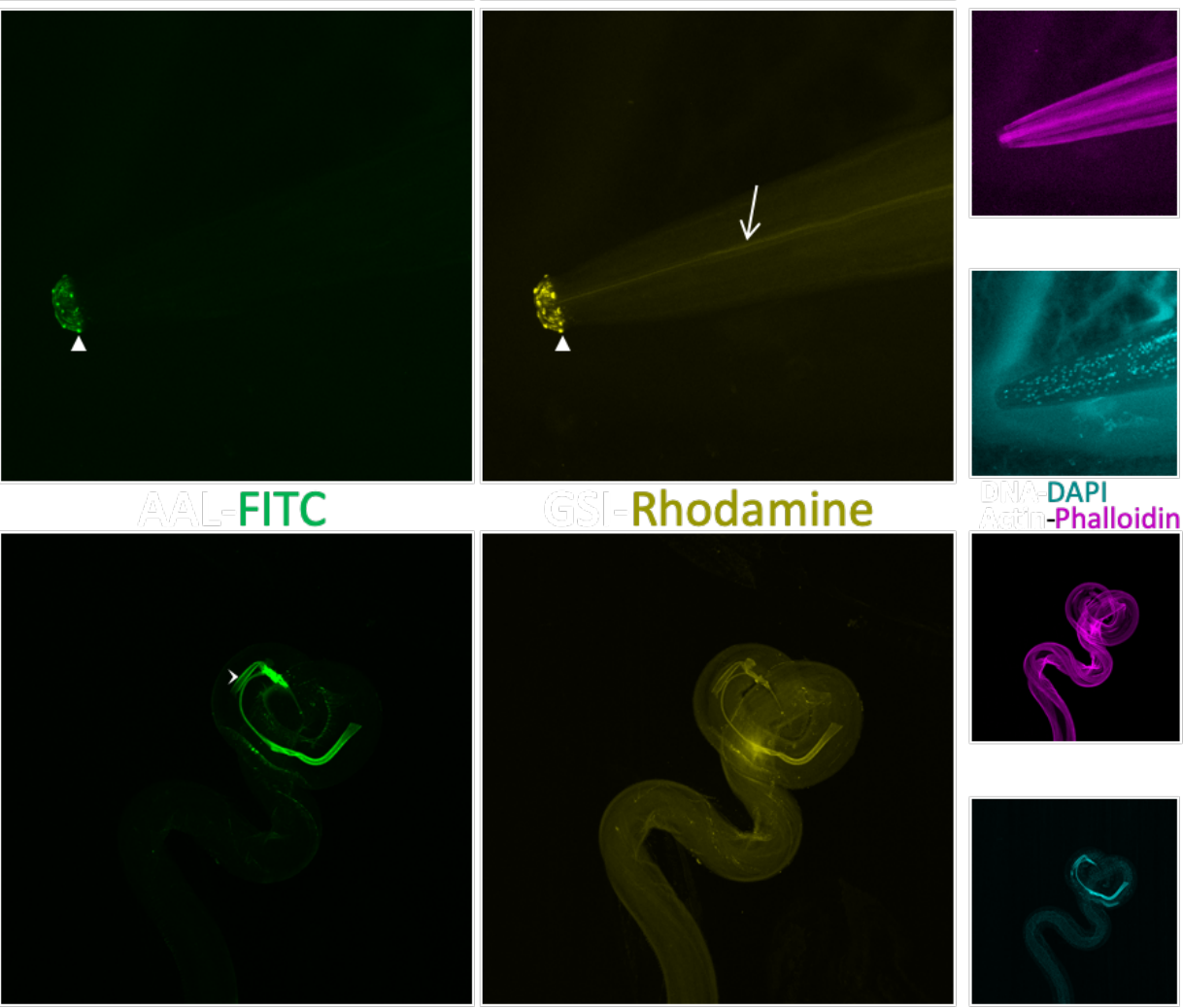

Male

tail
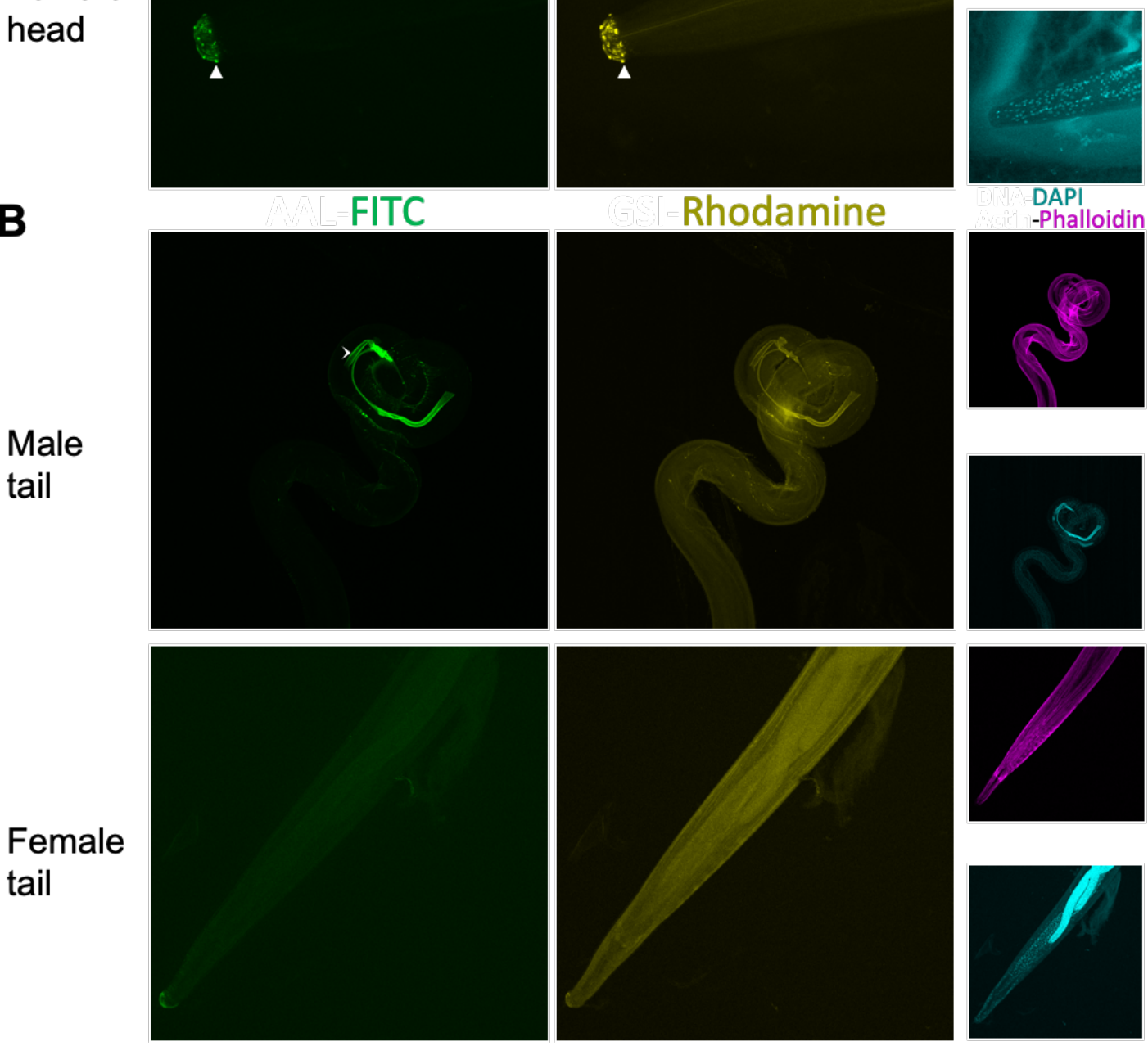

DAPI
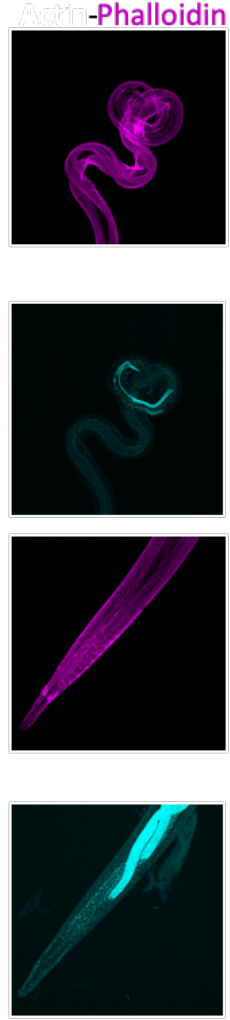

Figure 5: Fluorescence microscopy of male and female $B$. malayi adult worm heads and tails stained with fluorophore-labeled lectins. A. Heads of adult male and female worms. B. Tails of adult male and female worms. Images show full adult worms fixed in 4\% formaldehyde 2:1 in heptane and stained with AAL-FITC for Fucosylated glycan epitopes (Green), GS1-Rhodamine for Galactosylated glycan epitopes (Yellow), Phalloidin for actin (Magenta) and DAPI for DNA (Cyan). Images are taken at 20x magnification for both males and females. Images shown are representative of multiple biological replicates $(\mathrm{N}=3)$. Arrow heads point to sensory organs in cephalic regions of both males and females, arrows highlight the intestines of both males and females and the pointy arrow indicates male spicules (reproductive organs). 


\section{Adult male and female worms differentially glycosylate expressed proteins}

The differential localization of galactosylated and fucosylated residues suggests such modifications occur on different proteins. To identify the protein partners of the sex-biased glycan epitopes, we carried out proteomic analysis on glycoproteins isolated with AAL and GS-I (Fig. 6A). Consistent with our findings from the lectin arrays, binding to AAL and GS-I showed higher affinity to male and female lysates, respectively (Fig. 4). Total protein extracts from both male and female worms were subjected to affinity chromatography on AAL and GSI crosslinked columns. Chromatography fractions were checked for quality by silver stained SDS-PAGE gels (Supp. Fig. 1). Eluted proteins and column washes were labeled by tandem mass tags (TMT) and mass spectrometry analysis was done to identify the significantly enriched proteins in each fraction.

Following statistical analysis and normalization (see Materials and Methods), we identified a total of 56 unique proteins significantly enriched in the eluate from either columns, out of 950 proteins identified by MS/MS (two-way ANOVA, p < 0.005) (Supp. Table S3a). Of these proteins, 17 were galactosylated (bound to GS-I) and 32 were fucosylated (bound to AAL) in male worms, while 16 were galactosylated and only 6 were fucosylated in female worms (Fig. 6B-D; Supp. Table S3b). Clear sex-specific glycosylation of proteins was observed, with only 3/56 proteins displaying AAL-binding fucosylated epitopes in both sexes. (Fig. 6F)

To further investigate this subset of differentially glycosylated proteins, we profiled them for GO term enrichments (Supp. Table S3c). We observed a significant enrichment in proteins in the extracellular region $(p-$ value $=0.0014)$. This finding, coupled to the detection of Bmamif-1, a known immunomodulatory protein at the host-parasite interface, led us to determine whether these differentially glycosylated proteins were part of the B. malayi ES. All 56 detected proteins were observed in the $B$. malayi secretome defined in this study, suggesting a potential role for differential glycosylation in parasite glycoprotein-mediated immunomodulation. 


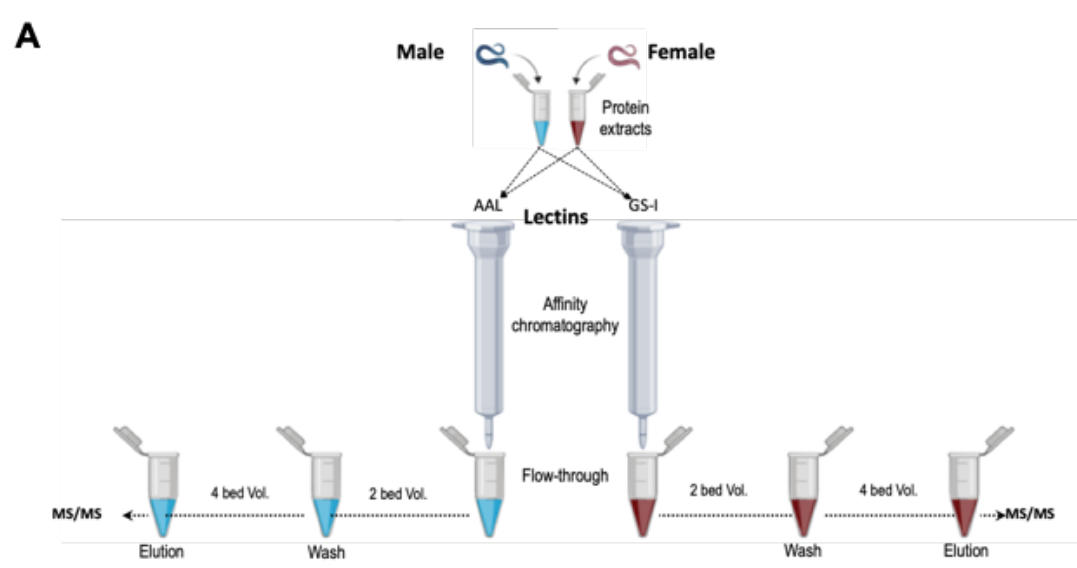

B

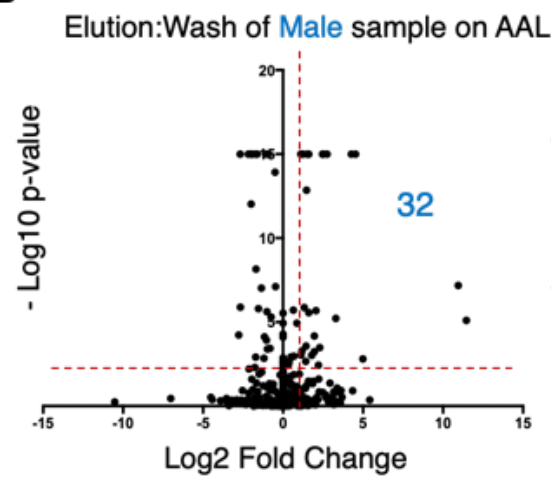

D

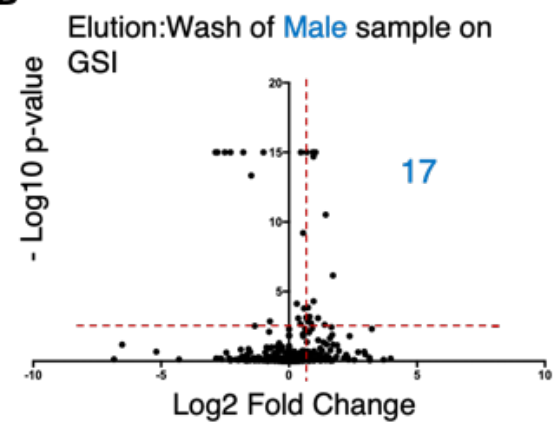

E

Elution:Wash of Female sample on GSI

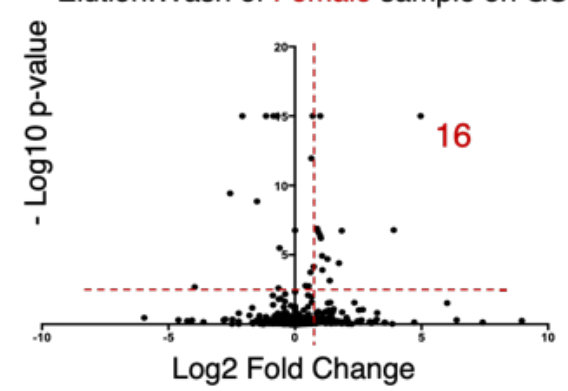

$\mathbf{F}$

\section{C}

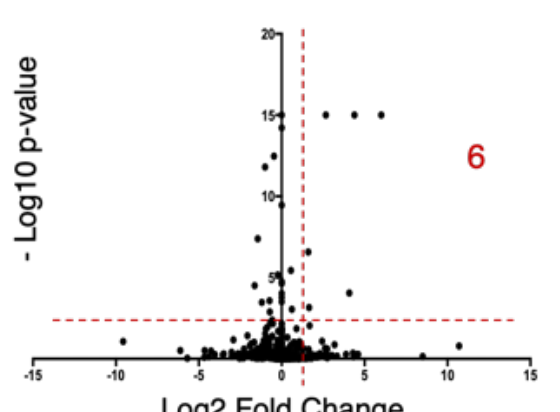

Log2 Fold Change

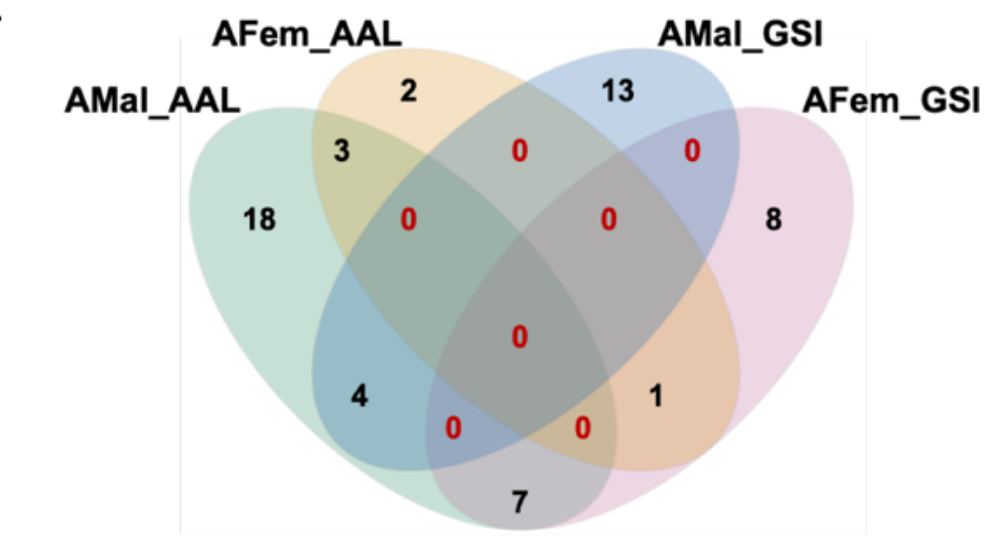



glycosylated proteins with potential immune functions

Four secreted B. malayi proteins have previously been reported to be immunomodulatory: cysteine protease inhibitor reported to be immunosuppressive; and the Cofactor Independent Phosphoglycerate Mutase Bma-IPGM-1 that induces a mixed Th1/Th2 response in the host

280 (Singh et al.).

282 To identify immunomodulatory candidates in $B$. malayi's secreted proteins, we made use of the guilt-by-association concept. We mined previously published B. malayi stage-specific transcriptomes for expression profiles of all ES protein coding genes and used dimensionality reduction (PCA, MDS) to identify genes that have similar expression profiles as the four known B. malayi immunomodulators (CPI2, MIF1, FAR1, and IPGM1). We clustered the results of both PCA and MDS analyses based on PC1:PC2 and X1:X2, respectively, to identify genes with the closest expression profile trends across all life stages. We then searched for known immunomodulators and identified the genes clustering with each of them. Both dimensionality reduction analyses and clustering (Supp. Figure 2) revealed a total of 112 proteins sharing similar expression profiles as the known immunomodulators, with more than $60 \%$ of those identified by both approaches (Fig. 7A and Supp. Table S4).

293 To further shortlist the candidates that are highly expressed and differentially glycosylated by 294 adult worms, we examined the overlap between the full set of differentially glycosylated proteins, the full set of in silico shortlisted candidates, and the top $50 \%$ secreted proteins detected in adult male and female worms. The analysis identified 16 proteins highly secreted

297 by both males and females and differentially glycosylated, including three of the known 298 immunomodulators secreted by B. malayi, Bma-MIF-1, Bma-FAR-2 and Bma-IPGM-1 (Fig. 
bioRxiv preprint doi: https://doi.org/10.1101/2021.02.24.432741; this version posted February 24, 2021. The copyright holder for this

preprint (which was not certified by peer review) is the author/funder, who has granted bioRxiv a license to display the preprint in perpetuity. It is made available under aCC-BY 4.0 International license.

299 7B). Gene identifiers, RNA expression levels, secreted protein levels, and individual function

300 annotations for each of the 12 proteins are provided (Fig. $7 \mathrm{C}$ ). 
A

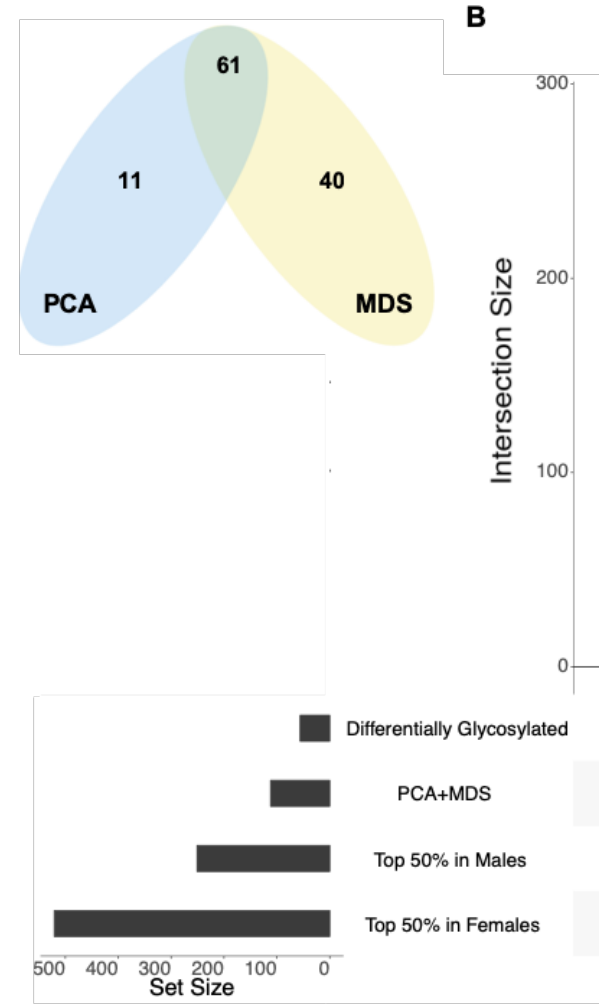

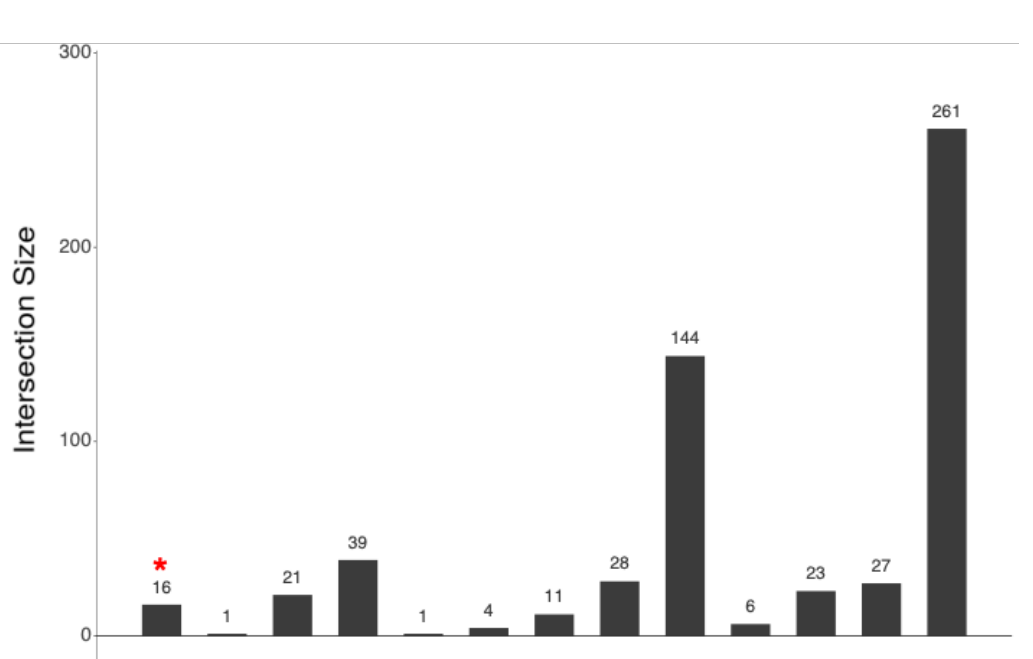

C

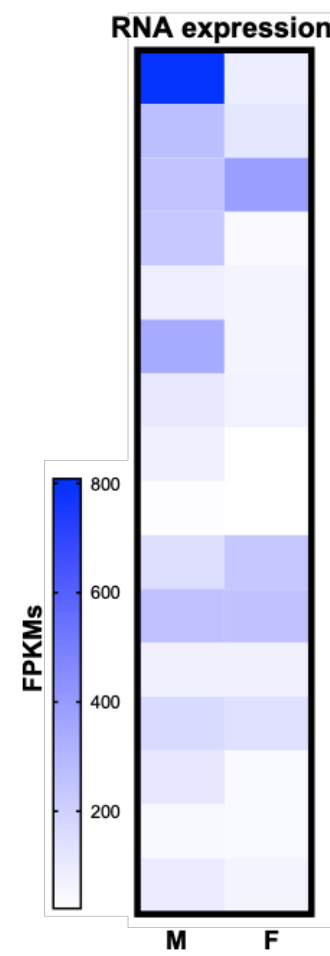

\begin{tabular}{ll} 
Gene name & \multicolumn{1}{c}{ Protein function } \\
Bm2151 & Glutathione peroxidase putative \\
Bm3952 & Fatty acid binding protein putative \\
$\underline{\text { Bm6870 }}$ & Bma-MIF-1; Macrophage migration inhibitory factor \\
\hline$\underline{B m 3146}$ & Bma-FAR-1; Fatty acid retinol-like binding protein \\
\hline Bm13317 & Bma-IPGM-1; cofactor independent phosphoglycerate mutase \\
Bm4709 & Bma-TR-51; TransThyretin-Related family domain \\
Bm5089 & Bma-CALU-1: Calumenin (calcium-binding protein) homolog \\
Bm2290 & Bma-DIM-1; Immunoglobulin-like putative \\
Bm3307 & Actin and ATP binding, putative \\
Bm2748 & GTP binding and GTPase activity, putative \\
Bm6327 & Bma-HSP-60; Heat Shock Protein- 60 \\
Bm1994 & Hydrolase, putative \\
Bm13661 & HSP70 putative \\
Bm2684 & TransThyretin-like, putative \\
Bm6198 & Bma-PCK-1; Phosphoenolpyruvate Carboxykinase \\
Bm13839 & Bma-PGK-1; Phosphoglycerate Kinase \\
&
\end{tabular}

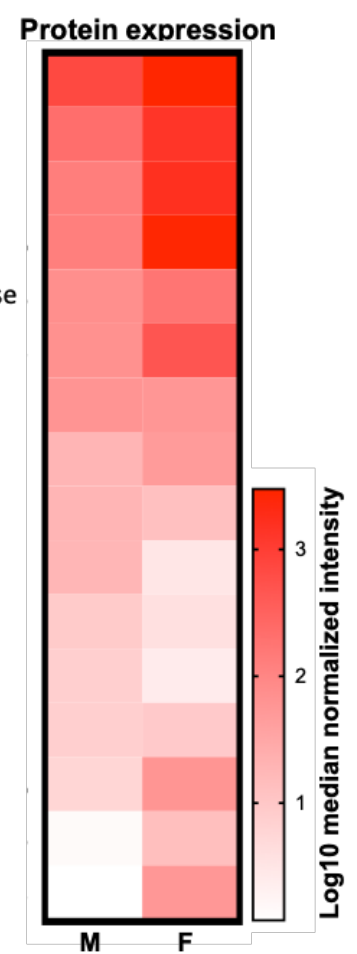

Figure 7: Immunomodulatory candidate proteins secreted and differentially glycosylated by adult male and female B. malayi. A. Venn diagram representing the overlap between immunomodulatory gene clusters following Principal Component Analysis (PCA) and Multi-Dimensional Scaling analysis (MDS) of stage and sex specific secretome transcriptomes. B. UpsetR plot representing the intersections across the candidate cluster genes, differentially glycosylated proteins and the top $50 \%$ proteins secreted from both Males and Females. C. Candidate proteins from intersections highlighted (*) in B. Gene names, protein functions and their corresponding RNA expression and protein intensity levels are shown. Highlighted by an underline are three previously characterized known immunomodulators. 


\section{Discussion}

305 Protein glycosylation is an evolutionarily conserved posttranslational modification that plays an important role in multiple biological processes, from early development to sexual maturity of many living organisms (Varki, Ajit; Cummings, R. D.; Esko, J. D.; Freeze, H. H.; Stanley,

308 P.; Bertozzi, C. R.; Hart, G. W.; Etzler and E.). The template-free synthesis of glycans leads

309 to a large diversity in glycan epitope structures. Several such glycoproteins at the host310 parasite interface in helminthic infections (McSorley et al.; Tundup et al.), including galectins, 311 are known to regulate host-parasite interactions (Baum et al.; Boscher et al.). Glycoproteins 312 also play crucial roles in sperm-egg biocommunication as well as immune signaling and 313 activation (Giovampaola et al.). Characterization of the glycome is therefore an important component to gain a better understanding of the underlying biology of helminthic parasitism.

315 In this study, we profiled the glycoproteome of $B$. malayi, a model for filarial parasites. By 316 analyzing male and female B. malayi transcriptomes separately for glycogenome expression, 317 and experimentally probing their respective glycomes by lectin microarrays, we identified clear 318 sex biases in glycogenome expression coupled to strikingly different lectin binding profiles

319 between adult male and female $B$. malayi. The transcriptomic data suggested sex biases in 320 glycan epitope expression, largely confirmed by lectin arrays.

321 Helminth glycomes are generally rich in oligomannose and paucimannose structures, 322 extensively fucosylated, including the highly immunoactive $\alpha 1-3$ fucosylation, and rich in 323 GlcNAc and GalNAc residues (Hokke and van Diepen). The lectin binding profiles show that 324 B. malayi is no exception, and additionally uncovers a sex-biased fucosylation of type 2 325 LacNAc (LN) in males while females favor $\alpha 1-2$ fucosylation. Helminthic glycans may be 326 branched with LacNAc and LacDiNAc residues, or otherwise truncated with simple terminal

327 GlcNAc or GalNAc. Both B. malayi sexes expressed enzymes needed to catalyze the addition 328 of $\beta 1,3-$ GalNAc in an O-linked manner to a Ser/Thr site forming the Tn antigen. The Tn antigen 
329 is a truncated glycan with terminal GalNac that is mostly known for its correlation with

330 promoting metastatic colorectal cancers (Liu et al.). A higher affinity to core 1 and Tn-antigen

331 binding lectins is reported in female worms as compared to male worms. This is partially

332 explained by the higher expression of the $ß-1,6-\mathrm{N}-$ Acetylglucosaminyltransferases (Bm3610

333 and Bm5268) by males, catalyzing further modifications of the Tn epitope to core 2 and core

3346 O-glycans. These epitopes are not detected by the core 1 O-glycan binding (AIA, MPA,

335 MNA-G) and Tn-binding (HAA, HPA) lectins which show enhanced binding to glycoproteins

336 from female worms.

338 To gain a better understanding of the sex bias in glycan epitope display, and identify any

339 functional implications, we determined the distribution and localization of differentially 340 expressed glycan epitopes in male and female worms. We observe the specific localization of 341 galactosylated and fucosylated epitopes in reproductive tissues, sensory organs, intestines

342 and cuticle. Such localization suggests a role for differential glycosylation in development, 343 reproduction, and at the host-parasite interface, with sex specificity. To further investigate the

344 functional implications of this differential glycosylation, we identified the protein partners of 345 these differentially displayed epitopes. Using lectin affinity chromatography coupled to mass 346 spectrometry we identified 56 differentially glycosylated proteins fucosylated and/or 347 galactosylated in a sex-specific manner by $B$. malayi. The overrepresentation of 348 secreted/excreted proteins amongst the differentially glycosylated proteins is not entirely 349 unexpected yet the $100 \%$ overlap between ES proteins, as characterized in our study, and the 350 differentially glycosylated proteins, strongly suggests a role for differential glycosylation in 351 parasite interactions with its host. Substantial overlap with known secreted proteins $(60 \%)$ 352 validated the results.

353 The missing proteins identified in other studies (Bennuru et al) but not in our study may be due to sample variation and technological limitations. Most strikingly, we observed that most secreted proteins were shared across developmental stages, while previous work reported a 
stage-specific secretome (Bennuru et al.). This implies that the number of stage-specific secreted proteins may be much smaller than previously thought.

358 One of the most interesting differentially glycosylated, highly secreted $B$. malayi proteins is the well characterized Macrophage Migration Inhibitory Factor 1 (MIF1, Bm6870), a known immunomodulatory protein secreted by B. malayi (Prieto-Lafuente et al.) and determined in our study to be exclusively fucosylated in male worms. Fucosylation has been linked to CD14dependent Toll Like Receptor 4 (TLR4) signaling activation (lijima et al.). LeX, a fucosecontaining glycan epitope, has also been implicated in mediating endocytosis of parasite glycoproteins to reach their effector sites. We hypothesize that fucosylation of MIF1 serves to decrease the immunogenicity of the parasite protein enhancing its suppressive function, or alternatively fucosylation may facilitate a higher internalization of the protein, similarly enhancing its suppressive function. An equally interesting differentially glycosylated protein is Bma-IPGM-1, previously shown to induce a mixed immune response, and which we showed displays a male-specific fucosylation coupled to female-specific galactosylation. These two well characterized B. malayi immunomodulators are joined by a third immunomodulatory protein Bma-FAR-1; two newly suggested drug targets, Bm5089 and Bm13839; and a subset of Heat Shock Proteins, Transthyretin-like proteins and a fatty-acid binding protein-all of which are part of protein families associated with host immunomodulation in helminthic infections, emphasizing possible sex-specific roles for B. malayi worms in the interactions with their hosts.

\section{Conclusion}

378 In this study we show that adult male B. malayi preferentially display higher levels of fucose, localize the bulk of fucosylated and galactosylated glycoproteins to highly metabolically active internal and external reproductive organs, and differentially glycosylate secreted and immunerelevant proteins. Adult females favor $\alpha 1-2$ fucosylation (previously reported to play key roles in sperm-egg biocommunication), extensively galactosylate proteins, and localize the bulk of

383 fucosylated and galactosylated glycoproteins to the intestines and secretory/excretory path 
384 with a clear signal from the anal pore and esophagus. Considering the respective localizations

385 of fucosylated and galactosylated glycoproteins, and the differential glycosylation of secreted

386 immunomodulatory and immune-relevant proteins, the data suggest that adult male and

387 female $B$. malayi worms may be modulating the host immune response in sex-specific

388 mechanisms and pathways. However, it remains to be determined if differential glycosylation

389 of secreted proteins alters their effect on host cells. The immune potential of recombinantly

390 expressed proteins with no glycosylation should first be established. The custom glycosylation

391 of these recombinantly expressed proteins, if feasible, could help elucidate the functional

392 implications of sex-dependent glycosylation of secreted proteins in B. malayi.

Materials and Methods

Worm culture and processing of the collected secretome

All B. malayi worms in this study were provided by the NIH/NIAID Filariasis Research Reagent Resource Center for distribution through BEI Resources, NIAID, NIH. A total of 30 adult males, 30 adult females, $300 \mathrm{~L} 3,300 \mathrm{~L} 4$ and $2 \times 10^{6}$ microfilariae (mf) were used to characterize their respective secretomes. Adults were cultured in pairs at 1 worm/ml of RPMI 1600 supplemented with $2 \%$ glucose. L3, L4 and mf were cultured in bulk in 5ml of RPMI1600 with $2 \%$ glucose.

404 Spent media for all cultures was collected every $24 \mathrm{hrs}$ and filtered through $0.22 \mu$ filters, aliquoted and snap-frozen in an ethanol/dry ice bath. Collection of spent media was done for 7 days and combined aliquots were then concentrated 100x using Amicon Ultra15 with a $3 \mathrm{Kda}$ cutoff (cat\# UFC900324) and buffer exchanged into cold sterile PBS, at pH 7.4. Concentrated samples were further split into 5 equal $100 \mu$ fractions and methanol-chloroform precipitation of proteins was performed. Briefly, $400 \mu \mathrm{l}$ of methanol was added to each $100 \mu \mathrm{l}$ of protein

410 sample and a subsequent $100 \mu$ l of chloroform was added on top. The mixture was then briefly 411 vortexed and $300 \mu \mathrm{l}$ of DI water added. Samples were then centrifuged at 13,000 rpm for 15 
412 mins. Tubes were left at room temperature for another 15 mins to achieve a clear phase

413 separation. The top layer was carefully removed leaving $50 \mu$ l of liquid. $400 \mu l$ of methanol

414 were then added and tubes tapped to induce mixing of phases. Samples were centrifuged as

415 before, and the methanol wash step was repeated twice. Tubes were subjected to speed

416 vacuum to evaporate all liquids and the resulting pellets were resolubilized using RapiGest SF

417 Surfactant according to manufacturer's protocol with a final concentration of $0.1 \%$ (RapiGest

418 SF, $1 \mathrm{mg}, 1 / \mathrm{pk}$ Part Number: 186001860). Samples were combined and analyzed by Mass

419 Spectrometry.

\section{Glycogenome characterization and ortholog analysis}

The full genome of $B$. malayi was downloaded from WormBase in 2018 and identified as B_malayi-4.0 assembly (Harris et al.).

426 Functional annotation: GO annotation of the genome was done using BLAST2GO v1.3.0 (Gö

427 Tz et al.). A full list of protein glycosylation related GO terms was then obtained through the 428 GO commission and can be found in Supp. Table S5a. The genome was filtered for genes associated with any of the glycosylation GO terms. A full list of 135 genes was identified and can be found in Supp. Table S5b. A similar analysis was done with the genome using the KEGG database (Kanehisa et al.) and all protein glycosylation related pathways can be found in Supp. Table S5c. Subsequently old gene identifiers from KEGG were assigned to the new IDs using BioMart for conversion (Smedley et al.). A total of 112 genes were found and are

434 listed in Supp. Table S5d. To complement the functional analysis, B. malayi's genome was analyzed using HMMER (Potter et al.) to annotate all Pfam domains. The full results are shown in Supp. Table S5e. Pfam annotations related to protein glycosylation were extracted from the Pfam database (Finn et al.); annotated genes can be found in Supp. Table S5f. 
439 BLASTp analysis: A full list of glycosylation related proteins in human was extracted from

440 Uniprot (D506-D515) and is shown in Supp. Table S5g. BLASTp was used to interrogate the

441 whole B. malayi proteome against the compiled "glycosylation proteome". Multiple blast hits

442 were allowed and filtered for a maximum e-value of 0.05 . All hits with at least $50 \%$ sequence

443 identity were considered true and Supp. Table S5h includes all data from the Blastp analysis.

444 Subsequently, hits with identity scores between $20-50 \%$ were further cross-checked against

445 KEGG, GO and Pfam outcomes and hits present in at least one of the functional annotations

446 were added. Supp. Table S5i shows a compiled list of unique genes per approach and the

447 overall unique list.

449 Ortholog analysis: To evaluate the conservation of all glycogenes across several other nematode genomes, Biomart (Smedley et al.) was used and all available genome projects for the following organisms were selected and analyzed for orthologs and their percentage identity. Organisms selected were Homo sapiens, C. elegans, F. hepatica, T. suis, O. volvulus,

\section{Transcriptome sequencing and analysis}

Lists of differentially expressed genes for male and female B. malayi were downloaded from

460 (Grote et al.) and further filtered for glycogenome expression; Supp. Table S2b represents 461 the full list of significantly differentially expressed glycosylation genes between adult male and female worms with corresponding functions. For dimensionality reduction, FPKM values used were generated as follows: BAM files were used with Cufflinks (v2.2.1) (Roberts et al.;

464 Trapnell, Hendrickson, et al.; Trapnell, Williams, et al.) to obtain fragments per kilobase of exon per million fragments mapped (FPKMs) for each of the annotated transcripts and with

466 Cuffnorm (Roberts et al.; Trapnell, Hendrickson, et al.; Trapnell, Williams, et al.) to obtain 
normalized FPKMs by library size. Data was filtered for secretome-coding genes as identified by our analysis. ClustVis (v1.0) (Metsalu and Vilo) was used for Principal Component Analysis and $\mathrm{R}$ was used to do Multi-Dimensional Scaling. PC1, PC2 and X1, X2 were then used for unsupervised clustering and plotting using the pHeatmap $\mathrm{R}$ package (https://cran.rproject.org/package=pheatmap, v 1.0.10).

\section{Protein extractions from male and female $B$. malayi adult worms}

Protein extractions from both male and female worms were done by cryogenic grinding and PBS extraction. Briefly, worms were ground for 2 cycles of 10 mins in liquid N2 with $100 \mu \mathrm{l}$ of PBS. The white powder was recovered and left to sit on ice until melted. Samples were then diluted 1:1 PBS and left overnight on an end-to-end rotator at $4^{\circ} \mathrm{C}$. The samples were centrifuged for 15 mins at $13,200 \mathrm{rpm}$ at $4^{\circ} \mathrm{C}$. Supernatants were collected, quantified and aliquoted for further use. A total of 500 male worms and 200 female worms were used, split into two batches for biological replication.

\section{Lectin arrays}

Lectin microarrays were generated as previously described (Pilobello, Krishnamoorthy, et al.).

Briefly, arrays were manufactured in-house with a Nano-plotter v2.0 piezoelectric non-contact array printer (GeSiM) using a nano A-J tip. They were printed on Nexterion Slide H (Schott Nexterion) under $50 \%$ relative humidity at a surface temperature of $12^{\circ} \mathrm{C}$. Commercial lectins and antibodies were purchased from Vector Labs, R\&D Systems, Santa Cruz, TCl, AbCam,

492 E.Y. Labs, or Sigma-Aldrich. The recombinant lectins rGRFT, rCVN, and rSVN were generous

493 gifts from Dr. B. O’Keefe (NCI Frederick). A list of all printed lectins can be found in Supp.

494 Table S6a. We note that we are unable to observe a subset of epitopes (e.g. $\alpha 2,8$-linked 
sialic acids) in our array. Prior to sample hybridization, lectin microarray slides were blocked

496 for $1 \mathrm{~h}$ with $50 \mathrm{mM}$ ethanolamine in $50 \mathrm{mM}$ sodium borate buffer ( $\mathrm{pH} 8.8)$ and washed three

497 times with $0.005 \%$ PBS-T ( $\mathrm{pH} 7.4$ ). Sample protein concentration and the degree of

498 fluorescent label incorporation was determined by measuring absorbances at 280,555 , and

$499650 \mathrm{~nm}$ per the manufacturer's instructions on a NanoDrop ND-2000c spectrophotometer

500 (Thermo Scientific). A total of $5 \mu \mathrm{g}$ of proteins per sample and contrasting labeled reference were mixed in $0.005 \%$ PBS-T $(\mathrm{pH} 7.4)$ for a final concentration of $100 \mathrm{ng} / \mu \mathrm{L}$ of protein. For reference, an equimolar mixture of all samples assayed was used. Slides were then loaded into a hybridization cassette (Arrayit) to isolate individual arrays (24 per slide). Samples were loaded onto individual arrays with a control array for the reference vs reference sample. Labeled protein samples were hybridized for $2 \mathrm{~h}$ at $25^{\circ} \mathrm{C}$ with gentle agitation. Following hybridization, samples were removed and arrays washed 4 times with $0.005 \%$ PBS-T (pH 7.4) for 10 minutes each. Slides were removed, submerged in $\mathrm{ddH} 2 \mathrm{O}$, and spun dry. Arrays were scanned using a GenePix 4300A array scanner (PMT 550 laser power 100\% for both fluorescent channels). Background-subtracted median fluorescence intensities were extracted using GenePix Pro v7.2. Nonactive lectins were defined as having an average of both channel

511 SNRs $90 \%$ of the data and removed prior to further analysis. Data were median-normalized in each fluorescent channel and the log2 of the sample/reference ratio was calculated for each technical replicate for each lectin. Technical replicates were then averaged for each lectin within each array. To identify significantly overrepresented glycan epitopes in male and female worms, a two-way ANOVA was done on the outcome of the lectin arrays and significant lectins and corresponding binding epitopes can be found in Supp. Table S6b.

521 AAL and GSI lectins crosslinked to agarose beads were acquired from Vector lab (Cat\# AL522 1393-2), and EY lab (Cat\# AK-2401-2) and packed into chromatography columns. 
523 Chromatography columns were used as per the manufacturer's protocol. Briefly, columns

524 were washed with $1.4 \mathrm{M} \mathrm{NaCl}$ solution prior to use and re-equilibrated with $\mathrm{PBS} \mathrm{pH}=7.4$ before

525 use. Samples were applied to the columns and flow-throughs collected by gravity flow.

526 Columns were washed with 2 bed volumes of PBS and wash fractions were collected. Elution

527 was done in 4 bed volumes with corresponding elution buffers (Glycoprotein Eluting Solution,

528 Cat. No. ES-3100 Vector labs for AAL column and 0.1M Melibiose monohydrate for GSI Cat\#

529 AK-2401-2). Eluted samples were further concentrated using Amicon Ultra15 3Kda (Cat\#

530 UFC900324) to a final volume of $500 \mu \mathrm{L}$ (10x concentration). Elution buffer was exchanged to

531 sterile cold PBS $\mathrm{pH}=7.4$ and samples were aliquoted and stored for further use. Progression

532 and quality of the chromatographies were assessed by SDS-PAGE silver-stained gels using

533 Pierce $^{\text {Tm }}$ Silver staining kit (ThermoFisher Scientific Cat\# 24612) and standard 15\% resolving

534 gel 30:1 Polyacrylamide: Bis acrylamide denaturing gels. Gels were run for $1.5 \mathrm{hrs}$ at $90 \mathrm{~V}$ in

$5351 \mathrm{x}$ Tris-Glycine-SDS and Precision Plus Protein ${ }^{\mathrm{TM}}$ Kaleidoscope ${ }^{\mathrm{TM}}$ Prestained Protein

536 Standards \#1610375 was used for reference.

Mass spectrometry analyses

541 To identify the proteins pulled down by the respective lectins, the last wash and the elution samples of each of the affinity chromatographies were subjected to mass spectrometry analysis. Similarly, we also analyzed resolubilized secretome samples. Samples were

544 prepared as follows: Briefly, we denatured the proteins by heating for $15 \mathrm{~min}$ at $90^{\circ} \mathrm{C}$. We added $1 \mu \mathrm{g}$ of mass spectrometry grade trypsin (Sigma Aldrich) and digested the proteins into peptides at $37^{\circ} \mathrm{C}$ overnight. For glycofractions, we measured resulting peptide concentrations

547 with the Pierce Quantitative Fluorometric Peptide Assay (ThermoFisher, \#90110); this step was not performed for the secretome samples. The glycofractions were labeled using TMT10plex Isobaric Label Reagent (ThermoFisher) while secretome samples were subjected 
551 C18 Spin Tips (Thermo Scientific, \#84850) per manufacturer's instructions. For both 552 experiments, we used an EASY-nLC 1000 coupled on-line to a q-Exactive HF spectrometer 553 (both Thermo Fisher Scientific). Buffer A (0.1\% FA in water) and buffer B (80\% acetonitrile, $554 \quad 0.5 \%$ acetic acid) were used as mobile phases for gradient separation. Separation was performed using a $50 \mathrm{~cm} \times 75 \mu \mathrm{m}$ i.d. PepMap C18 column (Thermo Fisher Scientific) packed with $2 \mu \mathrm{m}, 100 \AA$ particles and heated at $55^{\circ} \mathrm{C}$. We used a 155 min segmented gradient of $0.1 \% \mathrm{FA}$ (solvent $\mathrm{A}$ ) and $80 \% \mathrm{ACN} 0.1 \% \mathrm{FA}$ (solvent $\mathrm{B}$ ) at a flow rate of $250 \mathrm{nl} / \mathrm{min}$ as follows: 2 to $5 \% \mathrm{~B}$ for $5 \mathrm{~min}, 5$ to $25 \% \mathrm{~B}$ for $110 \mathrm{~min}, 25-40 \%$ B for $25 \mathrm{~min}, 49-80 \%$ B for $5 \mathrm{~min}$ and $80-95 \%$ B for 5 min. Solvent B was held at $95 \%$ for another 5 min.

560 For label-free analysis of the secretome, the full MS scans were acquired with a resolution of 120,000 , an AGC target of $3 \times 10^{6}$, with a maximum ion time of $100 \mathrm{~ms}$, and scan range of 375 to $1500 \mathrm{~m} / \mathrm{z}$. Following each full MS scan, data-dependent high-resolution HCD MS/MS spectra were acquired with a resolution of 30,000 , AGC target of $2 \times 10^{5}$, maximum ion time of $150 \mathrm{~ms}, 1.5 \mathrm{~m} / \mathrm{z}$ isolation window, fixed first mass of $100 \mathrm{~m} / \mathrm{z}$ and NCE of 27 with centroid mode.

566 For the TMT labeled samples of the glycofractions, the full MS scans were acquired with a resolution of 120,000 , an AGC target of $3 \mathrm{e} 6$, with a maximum ion time of $100 \mathrm{~ms}$, and scan range of 375 to $1500 \mathrm{~m} / \mathrm{z}$. Following each full MS scan, data-dependent high-resolution HCD MS/MS spectra were acquired with a resolution of 60,000 , AGC target of $2 \mathrm{e} 5$, maximum ion time of $100 \mathrm{~ms}, 1.2 \mathrm{~m} / \mathrm{z}$ isolation window, fixed first mass of $100 \mathrm{~m} / \mathrm{z}$ and NCE of 35 with centroid mode.

572 The RAW data files were processed using MaxQuant (version 1.6.1.0) to identify and quantify 573 protein and peptide abundances. The spectra were matched against the Brugia malayi Uniprot database (downloaded August 18, 2018) with standard settings for peptide and protein identification, that allowed for $10 \mathrm{ppm}$ tolerance, a posterior global false discovery rate (FDR)

576 of $1 \%$ based on the reverse sequence of the mouse FASTA file, and up to two missed trypsin 577 cleavages. We estimated protein abundance using iBAQ 3 for label-free experiments. 
578 TMT labeled data was then filtered for proteins recovered only in both replicas for each sample

579 type. Data is shown in Supp. Table S3a. Ratios of Elution:Wash and Wash:Elution were

580 calculated for each detected protein by sample. Two-way ANOVA was performed using Prism

$581 \quad v 7$ to identify significantly pulled down proteins in the eluted fractions as compared to their wash counterparts. An adjusted p-value cutoff of 0.05 and a minimum 2-fold change was used and a list of significantly eluted proteins can be found in Supp. Table S3b. Label-free data relevant to stage and sex-specific secretomes were normalized by total intensities and median normalized by sample. Raw IBAQ values for all replicates and proteins are available in Supp. Table S1a.

\section{Lectin staining and confocal microscopy}

Adult male and female worms were individually washed in PBS in a 12-well plate with $1 \mathrm{ml}$ of PBS per well. Worms were fixed and permeabilized in 4\% paraformaldehyde 2:1 in heptane and left on high intensity shaking for 30 mins at room temperature. Fixed worms were washed three times in PBS for 5 mins and transferred to new 12-well plates with $1 \mathrm{ml}$ per well of $1 \mathrm{x}$ Phalloidin-iFluor 647 Reagent (Abcam cat\# ab176759) in PBS pH=7.4 for 90 mins at room temperature with moderate shaking. Worms were washed in PBS three times for 5 mins and transferred to new 12 well plates with $1 \mathrm{ml}$ of Fluorescein labeled Aleuria Aurantia Lectin (Vector labs cat\# FL-1391) and Rhodamine labeled Griffonia Simplicifolia Lectin I (Vector labs cat\# RL-1102) in PBS $\mathrm{pH} 7.4$ with $0.2 \mathrm{mM} \mathrm{CaCl}_{2}$ at $10 \mu \mathrm{g} / \mathrm{ml}$ final concentrations for both labeled lectins. Plates were left at $4^{0} \mathrm{C}$ overnight on moderate shaking and following that washed three times in PBS and transferred to new 12-well plates with fresh PBS. Unstained controls were processed similarly without the addition of the labeled lectins. Stained and control worms were mounted on microscopy slides in VECTASHIELD® Hardset ${ }^{\mathrm{TM}}$ Antifade

604 Mounting Medium with DAPI (Vector labs cat\# H-1500) and left for 30 mins at room 605 temperature before storing at $4^{0} \mathrm{C}$ until imaging. 
606 All worms were imaged using a LSM Zeiss 880 confocal microscope with 20X air objective

607 using the following settings: $1024 \times 1024$ pixels with $1.0 \mu \mathrm{m} z$ stack step size, 8bit, 1.3 zoom.

608 High magnification cut-outs were imaged using the 100X oil objective using $0.29 \mu \mathrm{m} \mathrm{z} \mathrm{stack}$

609 step size and all other settings identical. Laser power was set at $1.2 \%(405 \mathrm{~nm}), 2 \%(488 \mathrm{~nm})$,

$6102 \%(561 \mathrm{~nm}), 4 \%(633 \mathrm{~nm})$. All tracks were imaged separately to minimize signal crosstalk.

611 Projections were constructed using the ZEN2012 software with the "Maximum Intensity

612 Projection" processing option. High magnification pictures were additionally filtered using the

613 "Median Filter" processing option with x/y kernel size set at 3 voxels.

614

\section{Data Access}

616 All raw glycomics data are made publicly available and can be found at 617 doi:10.7303/syn24862046. The mass spectrometry proteomics data have been deposited to 618 the ProteomeXchange Consortium via the PRIDE partner repository with the dataset identifier 619 PXD024252.

\section{Acknowledgments}

623 The authors would like to thank the NYUAD global PhD fellowship fund and the NYU Abu

624 Dhabi Faculty Research Fund AD060 for supporting this work. This research was supported by funding from the Canada Excellence Research Chairs Program (LM). This work was also supported in part by the Division of Intramural Research (DIR) of the NIAID/NIH (EG). 
bioRxiv preprint doi: https://doi.org/10.1101/2021.02.24.432741; this version posted February 24, 2021. The copyright holder for this

preprint (which was not certified by peer review) is the author/funder, who has granted bioRxiv a license to display the preprint in perpetuity. It is made available under aCC-BY 4.0 International license.

634 


\section{References}

Agrawal, Praveen, et al. "A Systems Biology Approach Identifies FUT8 as a Driver of Melanoma Metastasis." Cancer Cell, vol. 31, no. 6, Cell Press, June 2017, pp. 804819.e7, doi:10.1016/j.ccell.2017.05.007.

Ahmed, Umul Kulthum, et al. "The Carbohydrate-Linked Phosphorylcholine of the Parasitic Nematode Product ES-62 Modulates Complement Activation." Journal of Biological Chemistry, vol. 291, no. 22, 2016, pp. 11939-53, doi:10.1074/jbc.M115.702746.

Baum, Linda G., et al. "Microbe-Host Interactions Are Positively and Negatively Regulated by Galectin-Glycan Interactions." Frontiers in Immunology, vol. 5, no. JUN, Frontiers Research Foundation, 2014, doi:10.3389/fimmu.2014.00284.

Bennuru, Sasisekhar, et al. "Brugia Malayi Excreted/Secreted Proteins at the Host/Parasite Interface: Stage- and Gender-Specific Proteomic Profiling." PLoS Neglected Tropical Diseases, vol. 3, no. 4, 2009, doi:10.1371/journal.pntd.0000410.

Boscher, Cecile, et al. "Glycosylation, Galectins and Cellular Signaling." Current Opinion in

Choi, Tae-Woo, et al. "Novel Findings of Anti-Filarial Drug Target and Structure-Based 19, no. 11, MDPI AG, Nov. 2018, p. 3579, doi:10.3390/ijms19113579.

Cvetkovic, Jelena, et al. "Glycans Expressed on Trichinella Spiralis Excretory-Secretory Nucleic Acids Research, vol. 47, 2019, doi:10.1093/nar/gky1049. 
Gemmill, Alan W., et al. "HOST IMMUNE STATUS DETERMINES SEXUALITY IN A PARASITIC NEMATODE." Evolution, vol. 51, no. 2, Society for the Study of Evolution, Apr. 1997, pp. 393-401, doi:10.1111/j.1558-5646.1997.tb02426.x.

Giovampaola, Cinzia Della, et al. “Alpha $(1,2)$ Fucosylated Glycoepitopes from Invertebrates

to Humans." Research \& Reviews: Journal of Zoological Sciences, vol. 4, no. 3,

Gö Tz, Stefan, et al. "High-Throughput Functional Annotation and Data Mining with the doi:10.1093/nar/gkn176.

Grote, Alexandra, et al. "Defining Brugia Malayi and Wolbachia Symbiosis by Stage-Specific Dual RNA-Seq." PLoS Neglected Tropical Diseases, vol. 11, no. 3, Public Library of Science, Mar. 2017, doi:10.1371/journal.pntd.0005357.

Harischandra, Hiruni, et al. "Profiling Extracellular Vesicle Release by the Filarial Nematode Science, Apr. 2018, p. e0006438, doi:10.1371/journal.pntd.0006438. 065X.2009.00799.x.

Harris, Todd W., et al. "WormBase: A Modern Model Organism Information Resource." Nucleic Acids Research, vol. 48, 2020, doi:10.1093/nar/gkz920.

Heindel, Daniel W., et al. "Glycomic Analysis of Host Response Reveals High Mannose as a Key Mediator of Influenza Severity." Proceedings of the National Academy of Sciences of the United States of America, vol. 117, no. 43, National Academy of Sciences, Oct. 2020, pp. 26926-35, doi:10.1073/pnas.2008203117. 
vol. 160, no. 1, Elsevier, July 2008, pp. 8-21, doi:10.1016/j.molbiopara.2008.02.007.

692 Hokke, Cornelis H., and Angela van Diepen. "Helminth Glycomics - Glycan Repertoires and Host-Parasite Interactions." Molecular and Biochemical Parasitology, vol. 215, Elsevier B.V., July 2017, pp. 47-57, doi:10.1016/j.molbiopara.2016.12.001.

lijima, Junko, et al. "Core Fucose Is Critical for CD14-Dependent Toll-like Receptor 4 doi:10.1093/glycob/cwx075.

Jiang, Daojun, Ben Wen Li, et al. "Localization of Gender-Regulated Gene Expression in the Filarial Nematode Brugia Malayi." International Journal for Parasitology, vol. 38, no. 5, Pergamon, Apr. 2008, pp. 503-12, doi:10.1016/j.jpara.2007.09.010.

Jiang, Daojun, James Malone, et al. "Multiplex Proteomics Analysis of Gender-Associated Proteins in Brugia Malayi." International Journal for Parasitology, vol. 42, no. 9, Aug. 2012, pp. 841-50, doi:10.1016/j.jpara.2012.06.004.

Jiménez-Castells, Carmen, et al. "Gender and Developmental Specific N-Glycomes of the

Kanehisa, Minoru, et al. "New Approach for Understanding Genome Variations in KEGG."

Nucleic Acids Research, vol. 47, 2019, doi:10.1093/nar/gky962.

Kashyap, Sudhanva S., et al. "Emodepside Has Sex-Dependent Immobilizing Effects on Porcine Parasite Oesophagostomum Dentatum." Biochimica et Biophysica Acta (BBA) General Subjects, vol. 1861, no. 2, Elsevier, Feb. 2017, pp. 418-30,

Kaushal, Nuzhat A., et al. "Brugia Malayi: Stage-Specific Expression of Carbohydrates Containing N-Acetyl-d-Glucosamine on the Sheathed Surfaces of Microfilariae." Experimental Parasitology, vol. 58, no. 2, 1984, pp. 182-87, doi:10.1016/00144894(84)90033-X.

718 Khoo, K. H., and Anne Dell. "Glycoconjugates from Parasitic Helminths: Structure Diversity 
and Immunobiological Implications." Adv Exp Med Biol, vol. 491, 2001, pp. 185-205, doi:10.1007/978-1-4615-1267-7_14.

Kumar, Ranjeet, et al. "Characterization of Filarial Phosphoglycerate Kinase." Biochimie, vol. 165, Elsevier B.V., Oct. 2019, pp. 258-66, doi:10.1016/j.biochi.2019.08.012.

Liu, Zhe, et al. "Tn Antigen Promotes Human Colorectal Cancer Metastasis via H-Ras Mediated Epithelial-Mesenchymal Transition Activation." Journal of Cellular and Molecular Medicine, vol. 23, no. 3, Blackwell Publishing Inc., Mar. 2019, pp. 2083-92, doi:10.1111/jcmm.14117.

Manoury, Bénédicte, et al. "Bm-CPI-2, a Cystatin Homolog Secreted by the Filarial Parasite Brugia Malayi, Inhibits Class II MHC-Restricted Antigen Processing." Current Biology, vol. 11, no. 6, Cell Press, Mar. 2001, pp. 447-51, doi:10.1016/S0960-9822(01)00118-X.

McSorley, Henry J., et al. "Immunomodulation by Helminth Parasites: Defining Mechanisms and Mediators." International Journal for Parasitology, vol. 43, no. 3-4, 2013, pp. 30110, doi:10.1016/j.jpara.2012.11.011.

Mersha, Fana B., et al. "Computational and Experimental Analysis of the doi:10.1371/journal.pone.0216849.

Metsalu, Tauno, and Jaak Vilo. "ClustVis: A Web Tool for Visualizing Clustering of Multivariate Data Using Principal Component Analysis and Heatmap." Web Server Issue Published Online, vol. 43, 2015, doi:10.1093/nar/gkv468.

Moreno, Yovany, and Timothy G. Geary. "Stage- and Gender-Specific Proteomic Analysis of Brugia Malayi Excretory-Secretory Products." PLoS Neglected Tropical Diseases, vol.

746 Nutman, Thomas B. Lymphatic Filariasis. Vol. 1, PUBLISHED BY IMPERIAL COLLEGE 
PRESS AND DISTRIBUTED BY WORLD SCIENTIFIC PUBLISHING CO., 2000, doi:10.1142/p048.

Pilobello, Kanoelani T., Deepika E. Slawek, et al. "A Ratiometric Lectin Microarray Approach to Analysis of the Dynamic Mammalian Glycome." Proceedings of the National Academy of Sciences of the United States of America, vol. 104, no. 28, National Academy of Sciences, July 2007, pp. 11534-39, doi:10.1073/pnas.0704954104.

Pilobello, Kanoelani T., Lakshmipriya Krishnamoorthy, et al. "Development of a Lectin

Potter, Simon C., et al. "HMMER Web Server: 2018 Update." Web Server Issue Published Online, vol. 46, 2018, doi:10.1093/nar/gky448.

Prasanphanich, Nina Salinger, et al. "Glycoconjugates in Host-Helminth Interactions."

Prieto-Lafuente, L., et al. "MIF Homologues from a Filarial Nematode Parasite Synergize with IL-4 to Induce Alternative Activation of Host Macrophages." Journal of Leukocyte Biology, vol. 85, no. 5, 2009, pp. 844-54, doi:10.1189/jlb.0808459.

Propheter, Daniel C., et al. "Fabrication of an Oriented Lectin Microarray." ChemBioChem, vol. 11, no. 9, May 2010, pp. 1203-07, doi:10.1002/cbic.201000106.

Roberts, Adam, et al. "Improving RNA-Seq Expression Estimates by Correcting for Fragment Bias." Genome Biology, vol. 12, no. 3, BioMed Central, Mar. 2011, p. R22, doi:10.1186/gb-2011-12-3-r22.

Rodríguez, Ernesto, et al. "Glycans from Fasciola Hepatica Modulate the Host Immune Response and TLR-Induced Maturation of Dendritic Cells." PLoS Neglected Tropical Diseases, vol. 9, no. 12, Public Library of Science, Dec. 2015, doi:10.1371/journal.pntd.0004234. 
Research, vol. 73, no. 6, Springer-Verlag, Nov. 1987, pp. 550-56, doi:10.1007/BF00535332.

Singh, Prashant K., et al. "Cofactor Independent Phosphoglycerate Mutase of Brugia Malayi Induces a Mixed Th1/Th2 Type Immune Response and Inhibits Larval Development in the Host." BioMed Research International, vol. 2014, Hindawi Publishing Corporation, 2014, doi:10.1155/2014/590281.

Smedley, Damian, et al. "The BioMart Community Portal: An Innovative Alternative to Large, Centralized Data Repositories." Ibounyamine Nabihoudine, vol. 43, 2015, doi:10.1093/nar/gkv350.

Tawill, Salah, et al. "Both Free-Living and Parasitic Nematodes Induce a Characteristic Th2 Response That Is Dependent on the Presence of Intact Glycans." Infection and Immunity, vol. 72, no. 1, Jan. 2004, pp. 398-407, doi:10.1128/IAI.72.1.398-407.2004.

Trapnell, Cole, David G. Hendrickson, et al. "Differential Analysis of Gene Regulation at Transcript Resolution with RNA-Seq." Nature Biotechnology, vol. 31, no. 1, Nature Publishing Group, Jan. 2013, pp. 46-53, doi:10.1038/nbt.2450.

Trapnell, Cole, Brian A. Williams, et al. "Transcript Assembly and Quantification by RNA-Seq Reveals Unannotated Transcripts and Isoform Switching during Cell Differentiation." doi:10.1038/nbt.1621.

Tundup, Smanla, et al. "Polarization of Host Immune Responses by Helminth-Expressed

van den Berg, Timo K., et al. "LacdiNAc-Glycans Constitute a Parasite Pattern for Galectindoi:10.4049/jimmunol.173.3.1902. 
804 Varki, Ajit; Cummings, R. D.; Esko, J. D.; Freeze, H. H.; Stanley, P.; Bertozzi, C. R.; Hart, G. W.; Etzler, M., and E. "Essentials of Glycobiology, 3rd Edition." Cold Spring Harbor (NY), edited by Ajit Varki et al., Cold Spring Harbor (NY): Cold Spring Harbor Laboratory Press, 2015, http://www.ncbi.nlm.nih.gov/pubmed/27010055\%0Ahttps://www.ncbi.nIm.nih.gov/books/ NBK310274/.

810 Wuhrer, Manfred, et al. "Gender-Specific Expression of Complex-Type N-Glycans in

811 Schistosomes." Glycobiology, vol. 16, no. 10, Narnia, Oct. 2006, pp. 991-1006, 812 doi:10.1093/glycob/cwl020.

813 Zhan, Bin, et al. "Ligand Binding Properties of Two Brugia Malayi Fatty Acid and Retinol

814 (FAR) Binding Proteins and Their Vaccine Efficacies against Challenge Infection in

815 Gerbils." PLOS Neglected Tropical Diseases, edited by Patrick J. Lammie, vol. 12, no.

816 10, Public Library of Science, Oct. 2018, p. e0006772,

817 doi:10.1371/journal.pntd.0006772.

818 
826 Supp. Figure S1: Silver-stained SDS-PAGE analysis for chromatography quality control. Molecular weight markers are shown, wash and elution fractions were loaded to evaluate the quality of the chromatography. A significant reduction in proteomic content is observed between the wash and elution fractions validating the chromatography outcomes.

830

Supp. Figure S2: Dimensionality reduction and clustering of the $B$. malayi secretome. A. MDS analysis of the B. malayi secretome coding gene expression across multiple life stages and sexes. Defined cluster represents the genes clustering with the known

834 immunomodulatory protein coding genes (Highlighted in Blue). A single cluster of genes is reported to include all four known candidates. A total number of 101 genes are part of the cluster. B. PCA analysis of the B. malayi secretome coding gene expression across multiple

837 life stages and sexes. Defined cluster represents the genes clustering with the known 838 immunomodulatory protein coding genes (Highlighted in Blue). Two separate clusters are 839 reported to include all four known candidates. Combined, both clusters include 72 genes 840 clustering with known candidates.

Supp. Table S1: Stage and sex-specific B. malayi secretome analysis.

843 Supp. Table S2: B. malayi glycotranscriptome analysis.

844 Supp. Table S3: Differential glycosylation comparative analysis between adult male and female B. malayi.

846 Supp. Table S4: Dimensionality reduction approaches to $B$. malayi ES transcriptome 847 for immunomodulatory candidate identification.

848 Supp. Table S5: B. malayi glycogenome characterization by multiple functional 849 annotation approaches.

850 Supp. Table S6: Lists of Lectins used for glycoprofiling of adult male and female $B$. 851 malayi and corresponding list of lectins displaying sex-specific differential binding. 